$$
\text { BNE } 63615
$$

\title{
Ionization Cooling in the Muon Collider
}

\author{
R.C. Fernow, J.C. Gallardo, H.G. Kirk \& R.B. Palmer \\ Brookhaven National Laboratory \\ Upton, NY 11973-5000 \\ J. Norem \\ Argonne National Laboratory \\ Argonne, IL 60439-4815 \\ D.V. Neuffer \& A. Van Ginneken \\ Fermi National Accelerator Laboratory \\ Batavia, IL 60510 \\ M. Green \\ Lawrence Berkeley National Laboratory \\ Berkeley, CA 94720 \\ D. Winn \\ Fairfield University \\ Fairfield, CT 06430-5195
}

A. Skrinsky

Budker Institute of Nuclear Physics

Novosibirsk, Russia 630090

October 1996

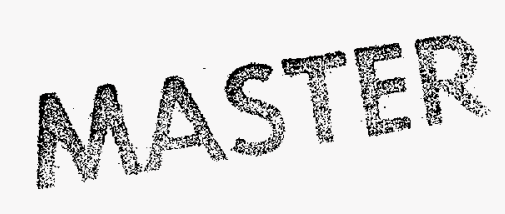

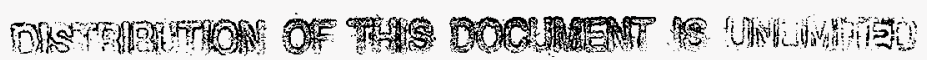




\begin{abstract}
The muon beams in a high luminosity muon collider are produced with a very large emittance. The process of ionization cooling offers a method for reducing the 6 dimensional normalized emittance of the beam by a factor of $\approx 10^{6}$. A simple analytic theory has been developed that demonstrates the dependence of the net cooling on various experimental parameters. The simple theory has been checked and realistic arrangements have been examined using Monte Carlo simulations. Transverse cooling of the initial beam can be achieved using passive Li absorbers in a FOFO lattice. The last factor of 10 in transverse cooling probably requires the use of current-carrying $\mathrm{Li}$ lenses. Efficient longitudinal cooling requires the use of wedge shaped absorbers in a dispersive section of the beam line. An example, multi-stage cooling scenario has been developed that meets the requirements of the muon collider. Preliminary designs have been made of solenoids for use in the FOFO lattice and of solenoids and dipoles for use in the emittance exchange sections. Detailed simulation work, further optimization, and preparations for experimental demonstrations of critical components are currently in progress.
\end{abstract}

\title{
1 Theory of Ionization Cooling
}

The possibility of building a muon-muon collider is currently the subject of active investigation[1]. In order to generate sufficient muons for the collider, it is necessary to capture a very large fraction of the pions created at the target. These pions, and the muons into which they decay, are then necessarily very diffuse (i.e. they have a very large emittance). In order to achieve the required luminosity of $10^{35} \mathrm{~cm}^{-2} \mathrm{~s}^{-1}$ at $2+2 \mathrm{TeV}$, it is necessary to reduce the transverse emittance in both the $\mathrm{x}$ and $\mathrm{y}$ dimensions by a factor of $\approx 300$ and the longitudinal emittance by a factor of $\approx 10$. This represents a reduction of the overall 6 -dimensional phase space by a factor of $\approx 10^{6}$. Therefore, it is essential to provide some means for cooling the muon beams.

The large mass of the muon compared to that of the electron prevents cooling by radiation damping, while the short lifetime of the muon prevents conventional stochastic or electron cooling. Fortunately, the process of ionization cooling can be used[1]-[4]. Because of their long interaction length this is possible only for muons. In this process the muon loses transverse and longitudinal momentum by $\mathrm{dE} / \mathrm{dx}$ in a material and then has the longitudinal momentum (but not the transverse momentum) restored in a subsequent $r f$ cavity. The combined effect is to reduce the beam divergence and thus the emittance of the beam. The process is complicated by the simultaneous presence of multiple scattering in the material, which acts as a source of heat and increases the emittance. The cooling effect can dominate for low $\mathrm{Z}$ materials in the presence of strong focussing fields. One solution being considered for the collider is to use absorbers made of lithium, beryllium, or liquid hydrogen inside a lattice of solenoid magnets. The absorber provides the energy loss, while the large aperture solenoids provide the required focussing.

The muons considered here lose energy primarily because of electromagnetic interactions with the atomic electrons. The rate of energy loss $-\mathrm{dE} / \mathrm{dx}$ is shown as a function of muon energy for several materials in Fig. 1. The energy loss falls dramatically as the particle energy increases from very small values. It reaches a minimum value for muons with energy 


\section{DISCLAIMER}

This report was prepared as an account of work sponsored by an agency of the United States Government. Neither the United States Government nor any agency thereof, nor any of their employees, make any warranty, express or implied, or assumes any legal liability or responsibility for the accuracy, completeness, or usefulness of any information, apparatus, product, or process disclosed, or represents that its use would not infringe privately owned rights. Reference herein to any specific commercial product, process, or service by trade name, trademark, manufacturer, or otherwise does not necessarily constitute or imply its endorsement, recommendation, or favoring by the United States Government or any agency thereof. The views and opinions of authors expressed herein do not necessar. ily state or reflect those of the United States Government or any agency thereof. 


\section{DISCLAMMER}

Portions of this document may be illegible in electronic image products. Images are produced from the best available original document. 


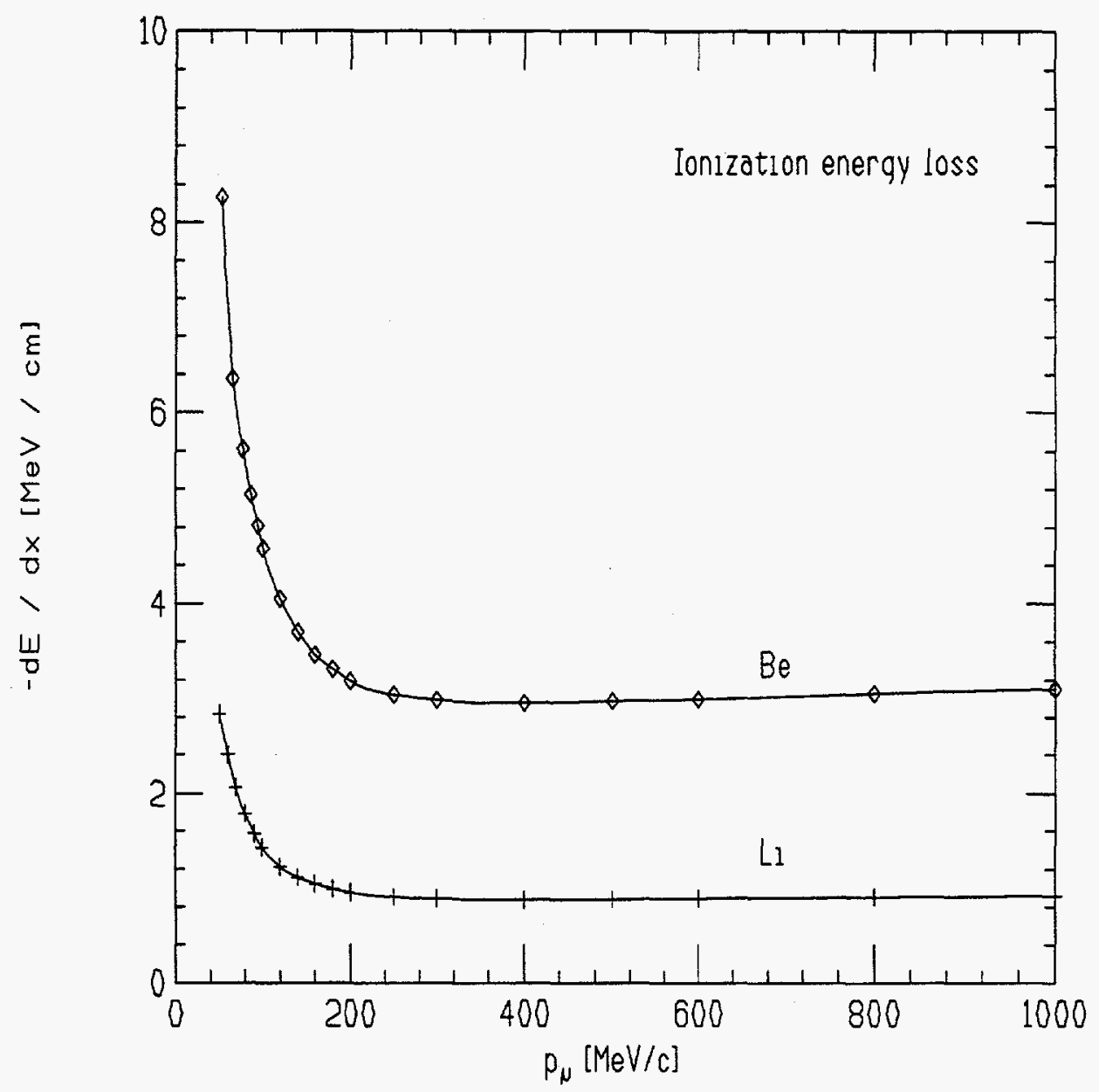

Figure 1: $\mathrm{dE} / \mathrm{dx}$ as a function of muon momentum for Li and Be.

around $300 \mathrm{MeV}$. Above this is the region of relativistic rise, where the energy loss increases very gently.

The basic principle illustrating ionization cooling of the transverse emittance is shown in Fig. 2. A diffuse beam of muons is focused onto a block of material. Muons travelling at an angle through the material lose both transverse and longitudinal components of momentum. Note that any part of the $\mathrm{dE} / \mathrm{dx}$ curve shown in Fig. 1 could be used for transverse cooling, although the focussing will be weaker at higher momentum. The beam then enters an accelerator cavity where the longitudinal momentum is returned to its starting value. However, since the transverse momentum is not replaced, the divergence of the beam is reduced. If the focusing and scattering properties of the material are such that the beam size does not increase, the geometric emittance of the beam is reduced by this process.

In order to cool the longitudinal emittance it must be arranged that the higher energy particles in the beam lose more energy than the lower energy particles. On the $\mathrm{dE} / \mathrm{dx}$ curve shown in Fig. 1 this only occurs for muon energies greater than $\approx 400 \mathrm{MeV}$. This natural longitudinal cooling is straightforward, but inefficient. A more practical idea, shown in Fig. 3, is to introduce dispersion into the beam, so that the muons receive a transverse 
displacement proportional to their deviation from the mean momentum. Then a wedge shaped absorber can be used to cause the higher momentum muons to lose more energy, and thus reduce the momentum spread in the beam.

\subsection{Transverse Emittance Cooling}

It will be useful to develop a simple model of emittance cooling in order to understand its dependence on various physical parameters, the optimum materials to use, and to estimate limits on the minimum achievable emittance. Let us adopt a coordinate system where $\mathrm{z}$ is along the direction of particle motion, and $\mathrm{x}$ and $\mathrm{y}$ are the orthogonal transverse directions. In the general case the geometric emittance is defined statistically as

$$
\left.\epsilon_{x}^{2}=\left\langle x^{2}\right\rangle\left\langle\theta^{2}\right\rangle-<x \theta\right\rangle^{2}
$$

where $\theta$ is the divergence angle of the particle trajectory projected onto the $\mathrm{x}-\mathrm{z}$ plane, and the expectation values are taken over all the particles in the beam. We define the normalized transverse emittance

$$
\epsilon_{x N}=\beta \gamma \epsilon_{x}
$$

\section{Ionization Cooling in Momentum Space}

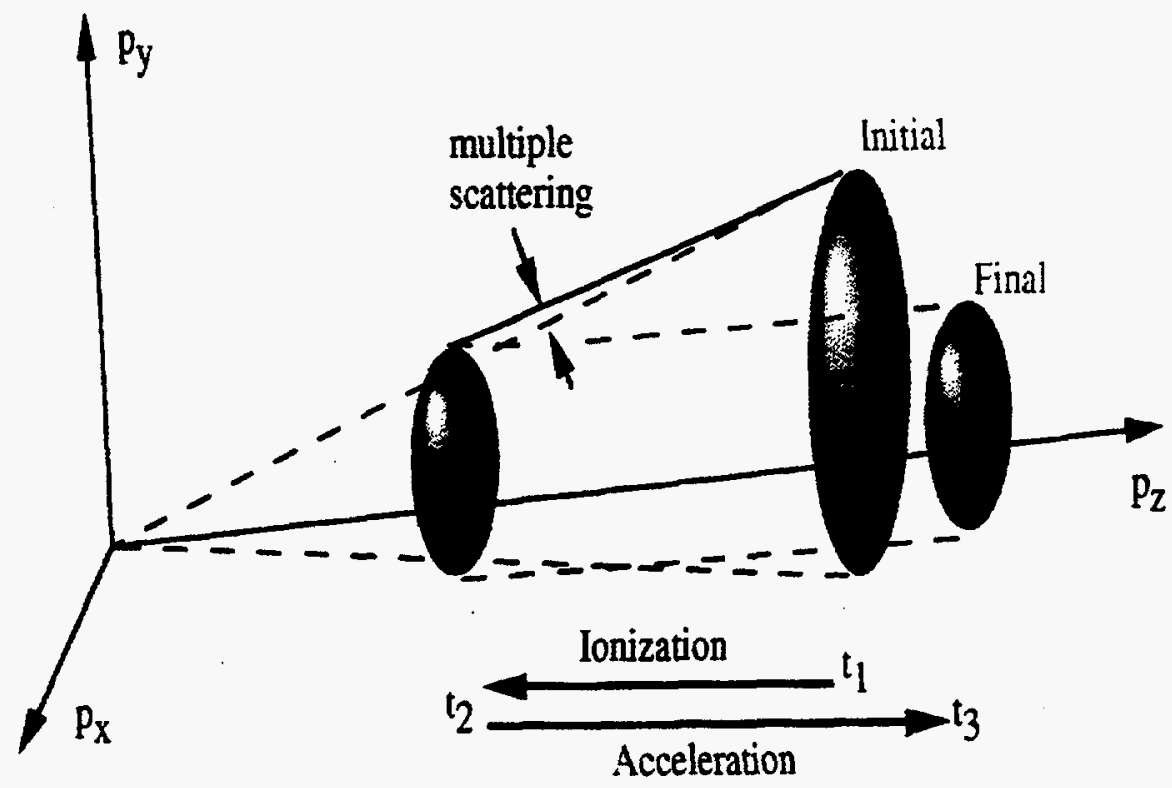

Figure 2: Basic principle of ionization cooling of transverse emittance. 
where $\beta, \gamma$ are the usual relativistic velocity and energy expressions. It is convenient to consider the change in the normalized emittance as the particles proceed through the absorbing material. Cooling of the normalized emittance takes place entirely in the absorber. The cooling arises because of the decrease of the $\beta \gamma$ factor in Eq. 2. Heating also occurs in the absorber, primarily because of multiple scattering. This will enter as an increase in the factor $\epsilon_{x}$ in Eq. 2. The subsequent accelerator section plays no role in this analysis, since the normalized emittance is unchanged by the acceleration process.

Now consider the change in $\epsilon_{x N}$ as the beam travels along the $\mathrm{z}$ direction into the material.

$$
\frac{d \epsilon_{x N}}{d z}=\epsilon_{x} \frac{d(\beta \gamma)}{d z}+\beta \gamma \frac{d \epsilon_{x}}{d z}
$$

It is possible to relate the first term in Eq. 3 to the ionization cooling

$$
\frac{d \epsilon_{x N}}{d z}(\operatorname{cool})=-\frac{1}{\beta^{2}} \frac{\epsilon_{x N}}{E}\left|\frac{d E}{d z}\right|
$$

where $E$ is the total energy of the muons. The second term in Eq. 3 gives rise to heating,

\section{USE WEDGE ABSORBER AT $\eta \neq 0$ TO INCREASE ENERGY.COOLING}

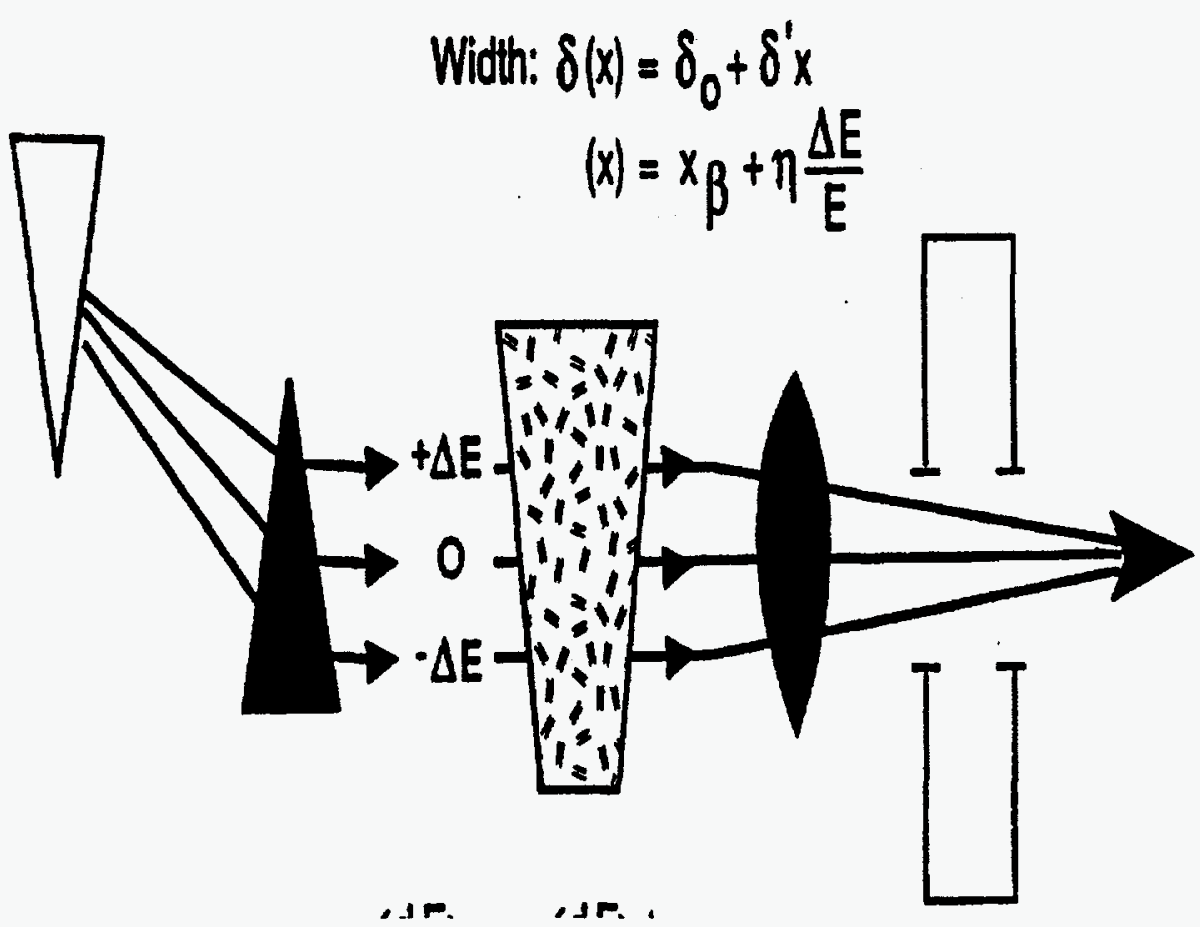

Figure 3: Basic principle of longitudinal cooling using a wedge absorber. 
which acts to increase the emittance as the beam proceeds through the material.

$$
\begin{aligned}
\frac{d \epsilon_{x N}}{d z}(\text { heat })= & \frac{\beta \gamma}{2 \epsilon_{x}}\left[<x^{2}>\frac{d}{d z}<\theta^{2}>+<\theta^{2}>\frac{d}{d z}<x^{2}>\right. \\
& \left.-2<x \theta>\frac{d}{d z}<x \theta>\right]
\end{aligned}
$$

At this point the simple theory derived above is exact, except for the neglect of additional processes such as muon bremsstrahlung and muon nuclear interactions that we assume are not important in the energy range under consideration.

Now let us assume that the cooling is taking place near a beam waist, so that we can neglect the effects of correlations in the beam parameters. If the focusing is sufficiently strong, we may also assume that the growth in the transverse size of the beam is negligible. It can be shown [5] that this will be the case, provided that the conditions

$$
\sigma_{x o}^{2} \gg \frac{\theta_{c}^{2} L}{2 \omega^{2}}
$$

and

$$
\sigma_{x o}^{2} \gg \frac{\theta_{c}^{2}}{4 \omega^{3}}
$$

are satisfied, where $\mathrm{L}$ is the length of the absorber material, $\omega$ is the focusing strength parameter $\left[\mathrm{m}^{-1}\right]$, and $\sigma_{x o}$ is the size of the beam entering the absorber. $\theta_{C}^{2}$ is a parameter $\left[\mathrm{m}^{-1}\right]$ related to the strength of the multiple scattering

$$
\theta_{C}=\frac{E_{S}}{p c \beta} \frac{1}{\sqrt{L_{R}}}
$$

where $\mathrm{E}_{S}=15 \mathrm{MeV}, \mathrm{p}$ is the particle's momentum, and $\mathrm{L}_{R}$ is the radiation length for the scattering medium. Thus, neglecting the second and third terms in Eq. 5, we find that

$$
\frac{d \epsilon_{x N}}{d z}(\text { heat }) \approx \frac{\beta \gamma}{2 \epsilon_{x}}<x^{2}>\frac{d}{d z}<\theta^{2}>
$$

If we use the relation

$$
<x^{2}>=\beta_{\perp} \epsilon_{x}
$$

from betatron focusing theory, we can write Eq. 9 in the form

$$
\frac{d \epsilon_{x N}}{d z}(h e a t) \approx \beta \gamma \frac{\beta_{\perp}}{2} \frac{d}{d z}<\theta^{2}>
$$

where $\beta_{\perp}$ is the betatron focusing parameter. The simplest expression for the change in $\theta^{2}$ can be found by using the Rossi-Greisen model[6] of multiple scattering

$$
\theta \approx \frac{E_{S}}{p c \beta} \sqrt{\frac{z}{L_{R}}}
$$


Although this expression is adequate for developing the simple scaling model, we use more accurate gaussian models and Moliere scattering theory in the Monte Carlo modelling described in section 2. With this approximation we can rewrite Eq. 11 as

$$
\frac{d \epsilon_{x N}}{d z}(\text { heat }) \approx \frac{\beta_{\perp}}{2} \frac{E_{S}^{2}}{\beta^{3} E m c^{2}} \frac{1}{L_{R}}
$$

where $\mathrm{m}$ is the mass of the muon.

We notice from Eqs. 4 and 13 that the rate of cooling decreases as the beam proceeds through the absorber, while the rate of heating increases. At some point the rates become equal and the absorber must end or the emittance will begin to increase again. If we consider the case when the two rates are equal, we find that the corresponding value of the emittance is

$$
\min \epsilon_{x N} \approx \frac{\beta_{\perp} E_{S}^{2}}{2 \beta m c^{2} L_{R}\left|\frac{d E}{d z}\right|}
$$

This is the minimum achievable emittance for the given material and focusing conditions. Note that the dependence on the focusing enters through the parameter $\beta_{\perp}$ in the numerator, while the dependence on the absorbing material enters through the product $L_{R} d E / d z$ in the denominator.

Table 1 gives the relevant parameters for some materials that might be useful for ionization cooling. The third column gives the energy loss for a minimum ionizing particle. The last column gives the coefficient of $\beta_{\perp}$ in Eq. 14 for a relativistic particle $(\beta=1)$. Thus, for example, if the focusing produces a value of $\beta_{\perp}=1 \mathrm{~cm}$, the estimated minimum emittance using Li would be $\approx 80 \mathrm{~mm} \mathrm{mr}$. The minimum achievable emittance would be a factor of 2 smaller if similar focusing could be used with liquid hydrogen.

Table 1: Materials for ionization cooling

\begin{tabular}{lcllc}
\hline \hline Material & $\rho$ & $\mathrm{dE} / \mathrm{dx}$ & $L_{R}$ & cof. of $\beta_{\perp}$ \\
\hline & {$\left[\mathrm{g} / \mathrm{cm}^{3}\right]$} & {$[\mathrm{MeV} / \mathrm{cm}]$} & {$[\mathrm{cm}]$} & {$[\mathrm{mm} \mathrm{mr} / \mathrm{cm}]$} \\
\hline liq. $\mathrm{H}_{2}$ & 0.071 & 0.286 & 890. & 42 \\
\hline liq. $\mathrm{He}$ & 0.125 & 0.242 & 756. & 59 \\
\hline $\mathrm{LiH}$ & 0.82 & 1.34 & 102. & 78 \\
\hline $\mathrm{Li}$ & 0.534 & 0.875 & 155. & 79 \\
\hline $\mathrm{Be}$ & 1.848 & 2.95 & 35.3 & 103 \\
\hline$: \mathrm{CH}_{2}:$ & 0.93 & 1.93 & 47.9 & 116 \\
\hline $\mathrm{C}$ & 2.265 & 3.95 & 18.8 & 144 \\
\hline liq. $\mathrm{N}_{2}$ & 0.807 & 1.47 & 47. & 155 \\
\hline $\mathrm{Al}$ & 2.70 & 4.36 & 8.9 & 275 \\
\hline \hline
\end{tabular}

It should be pointed out that transverse ionization cooling is always accompanied by some heating of the longitudinal emittance. This arises because ionization energy loss is a statistical process and there is a spread of energy losses (straggling) around the mean value. The design of the optimized cooling system reduces 6-dimensional phase space, as discussed in section 6.3 . 
In an alternate approach to estimating the equilibrium emittance, Skrinsky and Parkhomchuk [2],[4] have considered the transverse damping rate arising from the "frictional force" due to the ionization energy loss $\mathrm{dE} / \mathrm{dz}$. They derived the expression

$$
\min \epsilon_{x N} \approx Z \frac{L_{C}}{L_{I}} \frac{m_{e}}{m} \beta_{\perp}
$$

where $\mathrm{L}_{C}$ is the Coulomb logarithm, $\mathrm{L}_{I}$ is the Bethe-Bloch logarithm, and $\mathrm{m}_{e}$ is the mass of the electron. This expression gives an equilibrium emittance of $120 \mathrm{~mm} \mathrm{mr}$ for Li with $\beta_{\perp}=1 \mathrm{~cm}$, compared with the value of 80 that we found with the previous method. This indicates the level of uncertainty in this type of analysis. Accurate predictions for the amount of emittance reduction are derived using the Monte Carlo calculations described in section 2 .

\subsection{Longitudinal Emittance Cooling}

We define the normalized longitudinal emittance as

$$
\epsilon_{z N}=\beta_{z} \gamma \delta \sigma_{z}
$$

where $\sigma_{z}$ is the bunch length,

$$
\delta=\frac{\sigma_{p_{z}}}{p_{z}}
$$

is the fractional momentum spread, and $\sigma_{p_{z}}$ is the r.m.s. momentum spread in the beam. Now consider the change in the emittance as we take a step dz into an absorber material

$$
\frac{d}{d z} \epsilon_{z N}=\beta \gamma \delta \frac{d}{d z} \sigma_{z}+\beta \gamma \sigma_{z} \frac{d}{d z} \delta+\delta \sigma_{z} \frac{d}{d z}(\beta \gamma)
$$

where we have assumed the motion is predominantly along $z$. For relativistic beams changes in the bunch length will be small, so we drop the first term in Eq. 18. Then, using relations among the relativistic variables, it is possible to rewrite Eq. 18 in the form

$$
\frac{d}{d z} \epsilon_{z N} \approx \frac{\beta \gamma \sigma_{z}}{p_{z}} \frac{d}{d z} \sigma_{p_{z}}
$$

We identify three effects that modify the energy spread in the beam. Firstly, because of the curvature of the $\mathrm{dE} / \mathrm{dx}$ curve shown in Fig. 1, particles with different energies lose different amounts of energy. The fractional loss in energy in the step dz gives

$$
\frac{d}{d z} \sigma_{p_{z}}=\frac{\sigma_{E}}{\beta c} \frac{d}{d E}\left(\frac{d E}{d z}\right)
$$

This term increases the energy spread for particle energies below minimum ionization and decreases it (cools) for energies above it. However, the rate of cooling is very small since the slope of the $\mathrm{dE} / \mathrm{dx}$ curve in the region of relativistic rise is small. For the materials listed in Table 1 the rate of change of $\mathrm{dE} / \mathrm{dx}$ over the energy range $600-800 \mathrm{MeV}$ varies from $0.41 \times 10^{-4} / \mathrm{cm}$ for liquid hydrogen to $4.46 \times 10^{-4} / \mathrm{cm}$ for aluminum, in roughly the reverse 
order as their efficiency for transverse cooling. A longitudinal cooling scheme based on this effect, which achieves the requirements of the muon collider, would be at least an order of magnitude longer than the scheme presented in section 3.

Statistical fluctuations in the energy loss over a fixed distance of material is known as straggling. This leads to a second effect, which increases the amount of energy spread[7]

$$
\frac{d}{d z} \sigma_{p_{z}}=\frac{K_{s}}{2 \beta c \sigma_{E}} \gamma^{2}\left(1-\frac{1}{2} \beta^{2}\right)
$$

The constant

$$
K_{s}=4 \pi\left(r_{e} m_{e} c^{2}\right)^{2} \frac{N_{A} Z \rho}{A}
$$

where $r_{e}$ is the classical radius of the electron, $m_{e}$ is the mass of the electron, $\mathrm{c}$ is the speed of light, $N_{A}$ is Avogadro's number, and $\{Z, \rho, A\}$ are the atomic number, density, atomic weight $\}$ of the material. Note that the growth in energy spread is proportional to $\gamma^{2}$, so cooling at low energies is preferred.

It is also possible to cool the beam longitudinally by placing a transverse variation in absorber density or thickness in a region of non-zero dispersion, as shown in Fig. 3. We refer to this case as using a wedge absorber. We can write the fractional change in momentum spread as

$$
\frac{d}{d z} \sigma_{p_{z}} \approx \frac{1}{\beta c} \frac{d E}{d z} \frac{\eta \delta}{\alpha L_{o}}
$$

where the dispersion $\eta=d x / d \delta$, the wedge angle $\alpha=d x / d z$, and $L_{0}$ is the thickness of the wedge at $\mathrm{x}=0$. The longitudinal cooling will be associated with heating in the transverse phase space due to multiple scattering in the absorber.

\subsection{Transport Equation Approach}

In the transport equation approach a differential equation is set up that describes the evolution of a distribution function for the variables of the theory, such as angle, position, and energy. Fernow and Gallardo[8] considered the distribution $W(y, \theta ; z)$ in transverse position $y$ and deflection angle $\theta$ for the case of constant energy and an external focusing force. They confirmed that the beam size remains constant for the case of a strong magnetic field and derived conditions for the validity of Eq. 13 for the increase in emittance due to heating.

Vsevolozhskaya[9] considered the transport equation for the more general case where energy loss and reacceleration are also considered. This approach combines changes in transverse and longitudinal phase space into a unified formalism. Consider a beam of muons traversing a cooling section consisting of blocks of absorber, accelerator sections, and external focusing. The function $P(r, \theta, E ; z) d^{2} r d \theta d E$ gives the distribution of particles in the beam that have transverse radius between $\mathrm{r}$ and $\mathrm{r}+\mathrm{dr}$, polar angle between $\theta$ and $\theta+d \theta$, and energy between $\mathrm{E}$ and $\mathrm{E}+\mathrm{dE}$ at a distance $\mathrm{z}$ along the section. Then $\mathrm{P}$ satisfies the transport equation

$$
\frac{\partial P}{\partial z}+\theta \frac{\partial P}{\partial r}+\left(\theta \frac{\xi_{o}}{p v}+k r\right) \frac{\partial P}{\partial \theta}-2 \xi_{o} \frac{P}{p v}+\left(\xi_{o}-\xi\right) \frac{\partial P}{\partial E}=\frac{E_{k}^{2}}{4 p^{2} v^{2} L_{R}} \frac{\partial^{2} P}{\partial \theta^{2}}
$$

where $\xi_{o}$ is $\mathrm{dE} / \mathrm{dx}$ due to acceleration, $\xi$ is $-\mathrm{dE} / \mathrm{dx}$ due to ionization energy loss, $\mathrm{k}$ is the strength of the external focusing, and $E_{k} \approx 20 \mathrm{MeV}$ is a characteristic scattering energy. 


\subsection{Comparison of Focusing Methods}

Ionization cooling requires some form of focusing to limit emittance growth due to an increase in the size of the beam. Particles in the beam undergo betatron oscillations given in a region of constant focusing strength by

$$
x(z)=\sqrt{\epsilon \beta_{\perp}} \cos \left(\frac{z}{\beta_{\perp}}-\psi_{o}\right)
$$

where $\psi_{o}$ is the phase advance. The focusing strength is defined as

$$
k\left[\mathrm{~m}^{-2}\right]=\frac{0.3 \mathrm{~g}[\mathrm{~T} / \mathrm{m}]}{p[\mathrm{GeV} / \mathrm{c}]}
$$

where $\mathrm{g}$ is the field gradient. The betatron parameter is related to the focusing strength by

$$
\beta_{\perp}=\frac{1}{\sqrt{k}}
$$

while the focal length of an element of length $\mathrm{L}$ is

$$
f \approx \frac{1}{k L}
$$

We have considered four possible focusing elements for a linear cooling section.

\section{Quadrupole FODO cell}

Quadrupoles can be made with gradients $\mathrm{g} \approx 2 \mathrm{~T} / \mathrm{cm}$. By its nature a quadrupole focuses in one transverse dimension and defocuses in the other. The FODO lattice consists of equal strength, horizontally and vertically focusing quadrupoles separated by a drift distance. A cooling channel inside a FODO (or FDO) lattice could be a suitable arrangement for a higher momentum muon beam, although this has not been studied in detail. Some disadvantages of a quadrupole focusing system is that at least a triplet of elements are required for symmetric focusing and the defocusing action of the first quadrupole reduces the angular acceptance of the lattice.

\section{Solenoid}

A solenoid produces a central longitudinal magnetic field given by

$$
B_{z}=\mu_{0} n I
$$

where $I$ is the current and $n$ is the number of turns per unit length. A particle travelling parallel to the axis of a solenoid at a radius $r_{0}$ receives an azimuthal momentum kick

$$
p_{\phi}=\frac{e B_{z} r_{o}}{2}
$$

while crossing the fringe field region at the end of the solenoid. This produces a radial force in the central part of the solenoid, causing the particle to follow a helical trajectory with radius of curvature

$$
R=\frac{r_{0}}{2}
$$


Thus the radius of a single particle oscillates between $r_{o}$ and the axis of the solenoid. The envelope of a matched beam, however, is constant inside the solenoid. A short solenoid can be used as a lens with focal length

$$
f \approx \frac{4 p^{2}}{e^{2} B^{2} L_{s}}
$$

It is also possible to confine the beam in a long solenoidal channel. The angle of rotation around the axis of the solenoid of a particle at a distance $\mathrm{z}$ along the central portion of the solenoid is given by

$$
\theta=\frac{e B_{z} z}{p_{z}}
$$

The betatron amplitude is given by

$$
\beta_{\perp}=\frac{2 p_{z}}{e B_{z}}
$$

These relations can be significantly altered if space charge effects in the beam are important[10].

\section{Solenoid FOFO cell}

The solenoid FOFO cell consists of a series of short solenoids, separated by a distance d. The system focuses both transverse planes simultaneously. We can determine the basic properties of the cell in the thin lens approximation in the usual way by comparing the transfer matrix for the cell with the standard form of the Courant-Snyder matrix[11]-[12]. The phase advance per cell is

$$
\cos \psi=1-\frac{d}{2 f_{s}}
$$

where $f_{s}$ is the focal length of an individual solenoid. The maximum value of the betatron amplitude occurs at the midplane of the solenoid

$$
\beta_{\max }=d \frac{\kappa}{\sqrt{\kappa-\frac{1}{4}}}
$$

where $\kappa=f_{s} / d$. For stable motion we must have $\kappa>1 / 4$, or $f_{s}>d / 4$. The minimum value occurs at the midpoint between the solenoids where

$$
\beta_{\text {min }}=d \sqrt{\kappa-\frac{1}{4}}
$$

The minimum momentum that can be stably transmitted through the FOFO lattice is

$$
p_{\min }=\frac{e B \sqrt{L_{s} d}}{4}
$$

\section{Lithium lens}

In the lithium lens an axial current produces an azimuthal magnetic field $B$ at a radius $r$ inside the lens given by

$$
B=\frac{\mu_{o} J r}{2}
$$


where $\mathrm{J}$ is the current density inside the lithium. Gradients $\approx 7 \mathrm{~T} / \mathrm{cm}$ have been achieved at FNAL[13]-[14] and the possibility of reaching gradients as high as $20 \mathrm{~T} / \mathrm{cm}$ has been considered[15]. The particle follow a helical orbit around the lens axis. This lens focuses both transverse planes simultaneously. The focusing strength is

$$
k=\frac{e \mu_{\mathrm{o}} J}{2 p}
$$

The betatron amplitude is

$$
\beta_{\perp}=\sqrt{\frac{p R}{0.3 B_{R}}}
$$

where $\mathrm{R}$ is the radius of the lithium rod and $B_{R}$ is the value of the magnetic field at $\mathrm{R}$. The largest incident angle of a trajectory that will be confined in the rod is

$$
\theta_{o}=\sqrt{\frac{0.3 R B_{R}}{p}}
$$

\subsection{Emittance Exchange}

Since transverse cooling of a moving beam is always associated with longitudinal heating and longitudinal cooling is always associated with transverse heating, it is useful to consider each of the steps in the cooling scenario from the point of view of emittance exchange. However, we should emphasize from the start that there is no Liouville theorem for 6-dimensional phase space conservation in the presence of materials and dissipative forces. The reduction in one phase space dimension and the increase in another one depend quantitatively on the actual configuration that is used. Fig. 4 shows a mixed phase space diagram where the transverse size of the beam is plotted along the horizontal axis and the relative momentum spread is plotted along the vertical axis. Consider an initially round beam in this phase space. If the beam enters a dispersive region, to lowest order, the beam size grows while the momentum spread remains constant. This produces the dotted ellipse in the figure. If the beam enters a wedge at this point, the momentum spread decreases while the size remains approximately constant. This produces the flattened upright ellipse in the figure. The important point is that this mechanism allows transverse and longitudinal emittance to be exchanged. For example, it is possible to cool more than necessary in one dimension and then exchange the cooling with another dimension, if that turns out to easier than directly cooling to the desired value.

\section{Monte Carlo Modelling of Ionization Cooling}

The analytic expressions developed in the previous section show the dependence of emittances on various quantities, such as the muon momentum and magnetic field strengths. This is useful for rough optimization of cooling system parameters. The expressions should also be suitable for estimating achievable emittances, hopefully to within a factor of 3 . However, this simple differential equation approach has a number of shortcomings:

- it only describes the average change in emittance 
- it cannot give the distribution of particles in phase space

- it cannot show any possible correlations between the transverse and longitudinal phase space variables

- it doesn't describe the loss of beam particles

- it doesn't include the effects of additional processes, such as incoherent nuclear scattering, bremsstrahlung, direct pair production, and nuclear interactions

- it does not include errors in the cooling elements, such as magnet strengths and positions, etc.

- it does not include space charge effects

For this reason, accurate numerical estimates of the achievable transverse and longitudinal emittances and the particle losses for a given cooling scenario must be obtained using Monte

dispersion followed by wedge leaving no dispersion

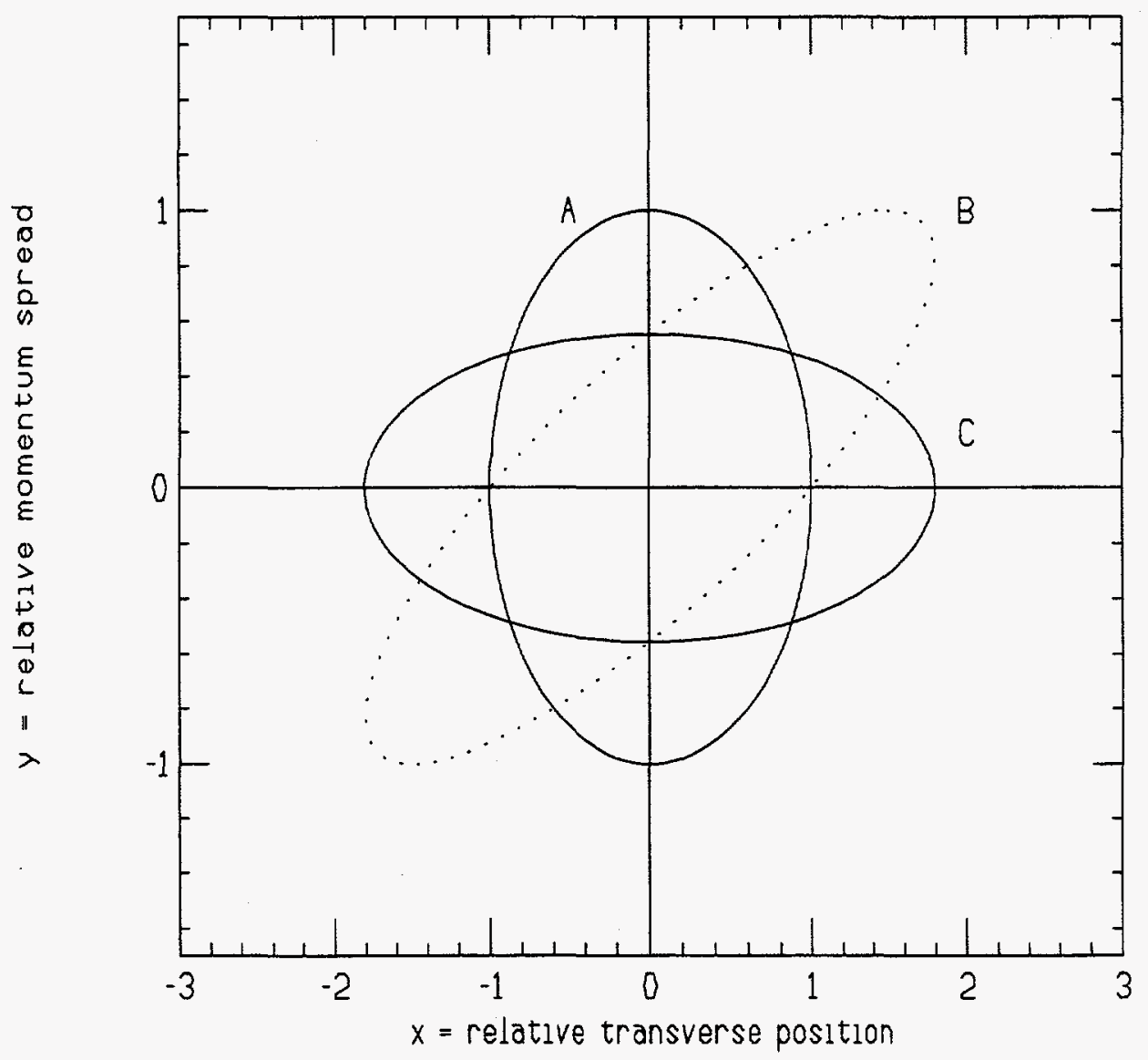

Figure 4: Emittance exchange diagram. Horizontal axis is transverse position; vertical axis is momentum spread 
Carlo simulations. An excellent overview of important issues in the simulation of ionization cooling has been given by Van Ginneken[16].

\subsection{Energy Loss and Multiple Scattering}

The most important processes for ionization cooling in the 10-300 MeV muon kinetic energy range are ionization energy loss off the atomic electrons and multiple Coulomb scattering off the nucleus of the absorber material. The mean rate of energy loss is described using the Bethe-Bloch formula. However, significant fluctuations (straggling) of the energy loss occur for each step through the material. The distribution of these fluctuations (Landau, Vavilov, or gaussian) are determined by the muon velocity and a parameter (described below) that is proportional to the step size. Fluctuations in the scattering angle after any step is given by the Moliere distribution. The actual distributions in energy loss and scattering angle are modified by the presence of the focusing magnetic field in the absorber material. In addition, the rarer processes described in the following section can introduce energy-angle correlations in the distributions.

The mean rate of ionization energy loss is given by the Bethe-Bloch equation[17]. A charged particle $(z=1)$ traversing matter loses energy at a rate

$$
-\frac{d E}{d z}=\frac{K Z \rho}{A \beta^{2}}\left[\frac{1}{2} \ln \frac{2 m_{e} c^{2} \beta^{2} \gamma^{2} T_{\max }}{I^{2}}-\beta^{2}-\frac{\delta}{2}\right]
$$

where $\{\mathrm{Z}, \mathrm{A}, \rho, \mathrm{I}\}$ are the atomic number, atomic weight, density, ionization potential $\}$ of the material, $\{\beta, \gamma, c\}$ are the usual relativistic factors for the incident particle, and $m_{e}$ is the electron rest mass. The constant $\mathrm{K}$ is given by

$$
K=4 \pi N_{A} r_{e}^{2} m_{e} c^{2}
$$

where $N_{A}$ is Avogadro's number and $r_{e}$ is the classical radius of the electron. The maximum possible energy transfer to the atomic electron is given by

$$
T_{\max }=\frac{2 m_{e} c^{2} \beta^{2} \gamma^{2}}{1+2 \gamma \frac{m_{e}}{M}+\left(\frac{m_{e}}{M}\right)^{2}}
$$

where $M$ is the mass of the incident particle. Fluctuations in the amount of energy loss are determined using the dimensionless ratio

$$
\kappa=\frac{\xi}{T_{\max }}
$$

where the characteristic energy

$$
\xi=\frac{2 \pi e^{4} N_{A} Z \rho}{m_{e} \beta^{2} c^{2} A} t
$$

is directly proportional to the spatial step size t. For $\kappa \leq 0.01$ the fluctuations can be described by the Landau distribution[18], provided[19] that the step is not allowed to be so small that the energy loss becomes comparable with the mean ionization potential I. Fig. 5 shows the Landau distribution for muons in $1 \mathrm{~cm}$ of $\mathrm{Li}$. The mean value of the energy loss is 


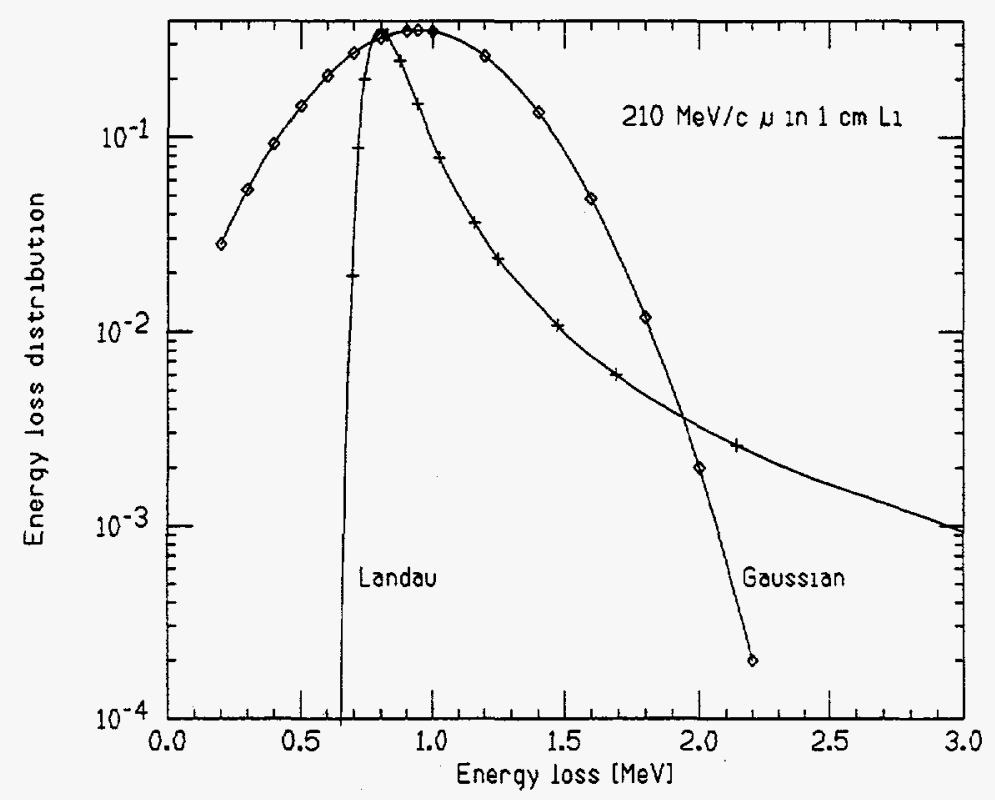

Figure 5: Landau distribution of energy loss in $\mathrm{Li}$

the same as that given by the Bethe-Bloch formula, although the most probable energy loss is smaller than the mean value. A gaussian distribution is shown on the same figure with a mean value given by the Bethe-Bloch formula and a standard deviation given by the Bohr formula (below).

For $0.01<\kappa<10$ energy loss is accurately described by the Vavilov distribution[20]. In addition to the value of $\kappa$, this distribution depends on the muon velocity. The lower limit of validity corresponds to characteristic energy losses on the order of the ionization potential.

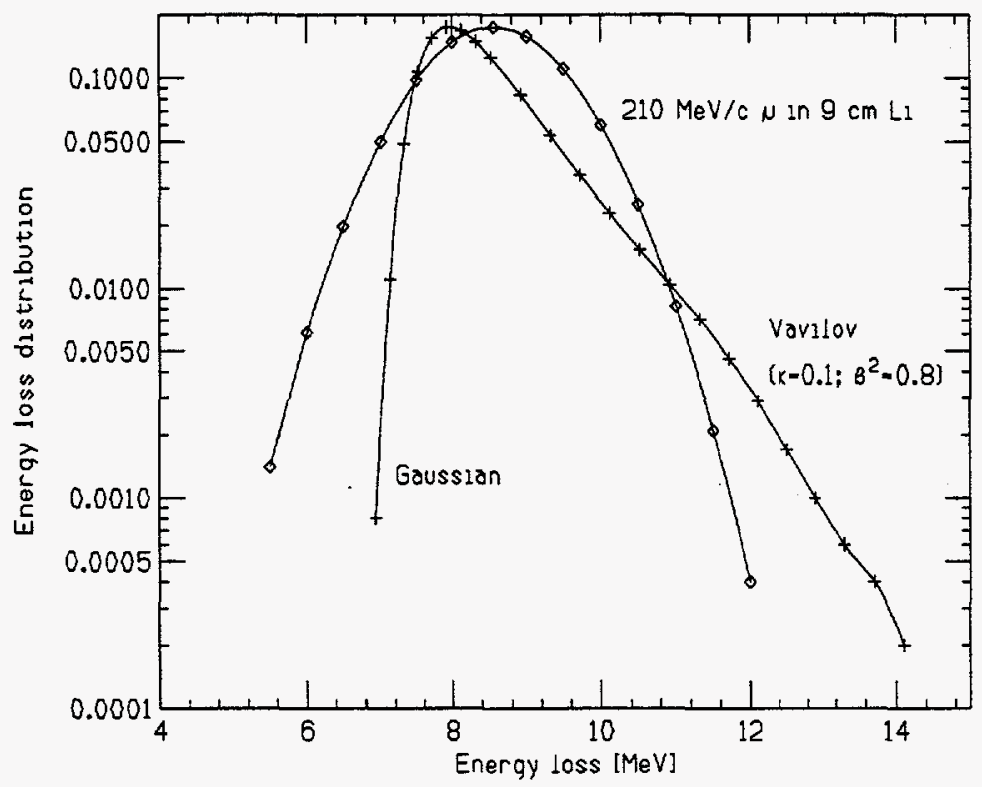

Figure 6: Vavilov distribution of energy loss in $\mathrm{Li}$ 
At very low values of $\kappa$ the Vavilov and Landau distributions coincide. Fig. 6 shows the Vavilov distribution for muons in a $9 \mathrm{~cm}$ step of Li. The gaussian distribution is obviously a better approximation here than for the $1 \mathrm{~cm}$ case. The Vavilov distribution was derived for a spin $J=0$ particle. Van Ginneken[16] has calculated a correction to the distribution for a spin $J=1 / 2$ particle, such as the muon, that causes a small increase in the width of the distributions.

A gaussian distribution accurately describes the energy loss when $\kappa \geq 10$. The variance of the distribution, first calculated by Bohr[7], is

$$
\sigma_{E}^{2}=4 \pi\left(r_{e} m_{e} c^{2}\right)^{2} N_{A} \frac{Z \rho}{A} \gamma^{2}\left(i-\frac{\beta^{2}}{2}\right) t
$$

Note that the variance grows quadratically with the energy $\gamma$ and linearly with the thickness t.

Coherent multiple Coulomb scattering of the muon from the nucleus of the absorber material is described by the Moliere distribution[21]. The characteristic scattering angle is

$$
\theta_{c}=\chi_{c} \sqrt{B}
$$

The parameter $\chi_{c}$ is determined from

$$
\chi_{c}^{2}=4 \pi\left(r_{e} m_{e} c^{2}\right)^{2} \frac{1}{(p c \beta)^{2}} \frac{N_{A} \rho Z(Z+1)}{A} t
$$

and is directly proportional to the thickness. The dimensionless quantity $B$ is determined from the equation

$$
B-\ln B=\ln \Omega_{0}
$$

where $\Omega_{o}$ is the mean number of collisions. Fig. 7 shows the Moliere distribution of scattering angles for muons crossing a $1 \mathrm{~cm}$ thickness of $\mathrm{Li}$. The figure also shows a gaussian distribution with standard deviation given by the Rossi formula, Eq. 12, with $E_{S}=21.2 \mathrm{MeV}$. The actual distribution of scattering angles is considerably wider than the gaussian approximation for angles greater than $\theta_{c}$.

The simulation code SIMUCOOL of Van Ginneken[16] introduces cut-off parameters for the energy loss and scattering angle. The corrected Vavilov distribution is used to simulate events below the cutoff energy loss on a statistical basis. Events with larger energy losses are treated as individual $\mu$ e scattering events using the Bhabha formula. The binding energy of the electron can be neglected for these relatively large losses. A given energy loss implies a unique scattering angle for the muon, thereby introducing energy-angle correlations into the distributions.

For Coulomb scattering between muons and nuclei the SIMUCOOL code introduces a cutoff angle at $2 \sigma_{\theta}$. Below this angle events are simulated statistically using a Gaussian distribution. Above it the Rutherford formula with a nuclear form factor is used to simulate individual events. Provided the cutoff is below the maximum angle as calculated on the basis of nuclear size, this should be a good approximation[22]. Note that here the Gaussian $\sigma$ is smaller than the unrestricted $\sigma_{\theta}$ given by Eq. 12. Again energy-angle correlations are 


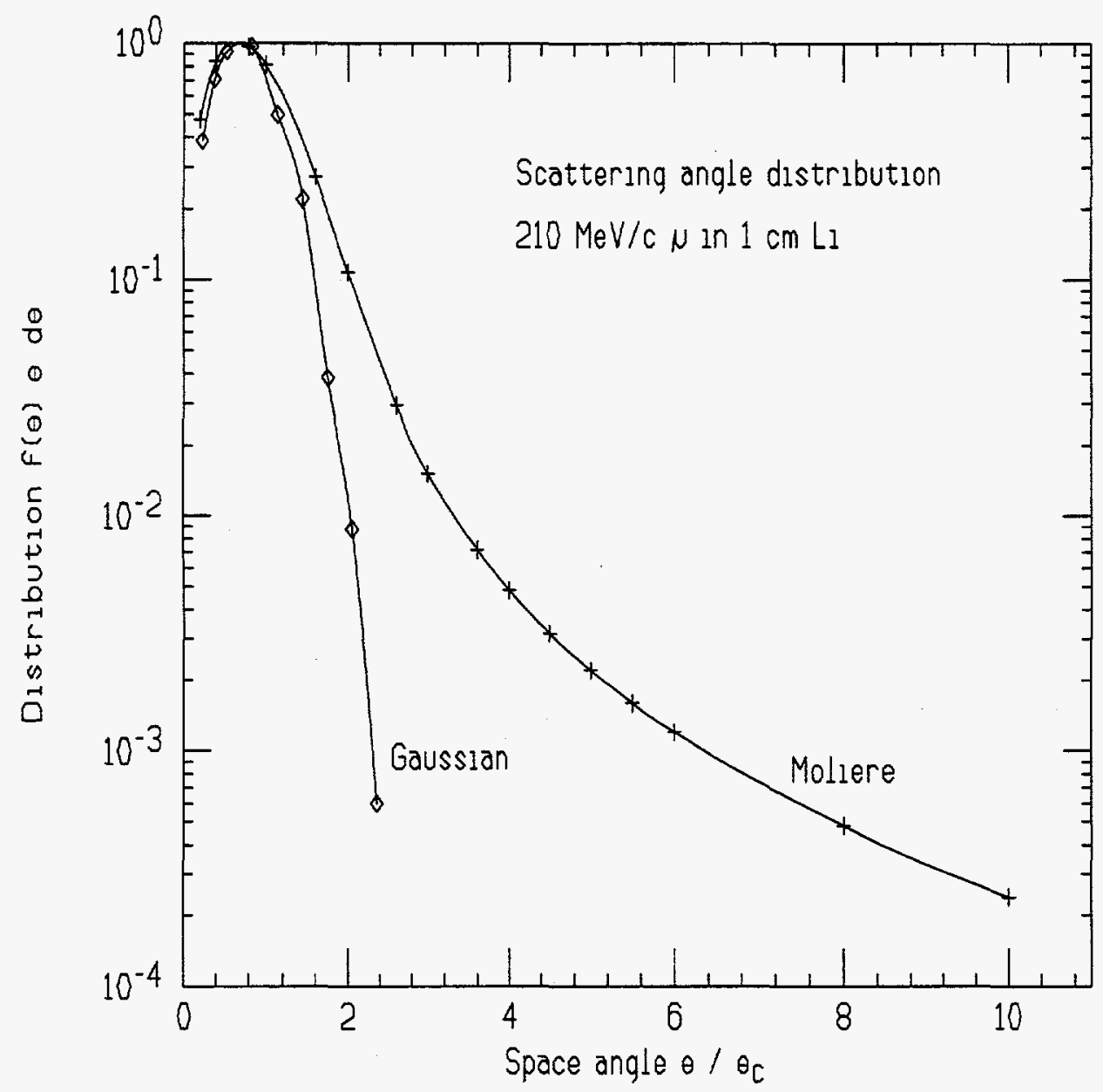

Figure 7: Moliere scattering angle distribution in $\mathrm{Li}$

introduced by applying the energy loss that accompanies the large angle events. Alternatively, codes based on the GEANT scattering algorithm (e.g. MUMC and ICOOL ) select a scattering angle from the Moliere scattering distribution whenever the mean number of expected scatters in a simulation step is larger than 20 . Otherwise, the actual number $\mathrm{N}$ of scatters is calculated from a Poisson distribution, and the scattering angle is determined from a series of $\mathrm{N}$ Rutherford scatterings.

\subsection{Other Relevant Effects}

We have seen in the previous section that the most important effects to simulate, as far as ionization cooling is concerned, are ionization energy loss and coherent multiple Coulomb scattering. Other effects play only a minor role in the ionization cooling process.

Muon decays are an important source of particle losses. However, since the decays are uncorrelated with the energy loss and scattering effects, they do not have a direct influence on the achievable muon emittances.

Van Ginneken has considered a number of other processes that only make a small contribution for $10-300 \mathrm{MeV}$ muons. These include bremsstrahlung, incoherent Coulomb 
scattering off individual protons in the nucleus, direct pair production, and deep inelastic nuclear interactions. Of these incoherent scattering may cause considerable angular deviation, whereas the others tend to produce relatively large energy loss. At low energy (where $\Delta E>T_{\max }$ ) $\mu$ e scattering becomes identically zero. For the low energy muons used in the cooling section of the muon collider, these processes only occur at very low levels:

\subsection{Tracking Simulations of Cooling}

Confirmation of ionization cooling using simulations of particle tracking has been made using at least five independent codes[23]. We present several examples here, as way of illustration.

Fig. 8 shows a SIMUCOOL simulation of transverse emittance cooling of $1 \mathrm{GeV}$ muons in a storage ring. The beam repeatedly crosses a $1 \mathrm{~cm}$ thick Be absorber in the ring. Each frame shows the transverse phase space at the traversal number shown in the lower right corner. The number in the upper left of each frame shows the number of muons remaining in the beam. Muons were removed from the simulation when $\mathrm{x}$ or $\mathrm{x}^{\prime}$ exceeded $3 \sigma$. The fractional reduction in phase space area is shown in the upper right corner. Thus in this example the transverse emittance is reduced to its asymptotic value after about 1500 turns. The emittance was reduced by a factor of 5 .

Modelling of cooling in a $1.5 \mathrm{~m}$ long $\mathrm{Li}$ rod has also been done using SIMUCOOL[24]. The rod had a radius of $10 \mathrm{~cm}$ and a $10 \mathrm{~T}$ surface field. The incident muon momentum was 400

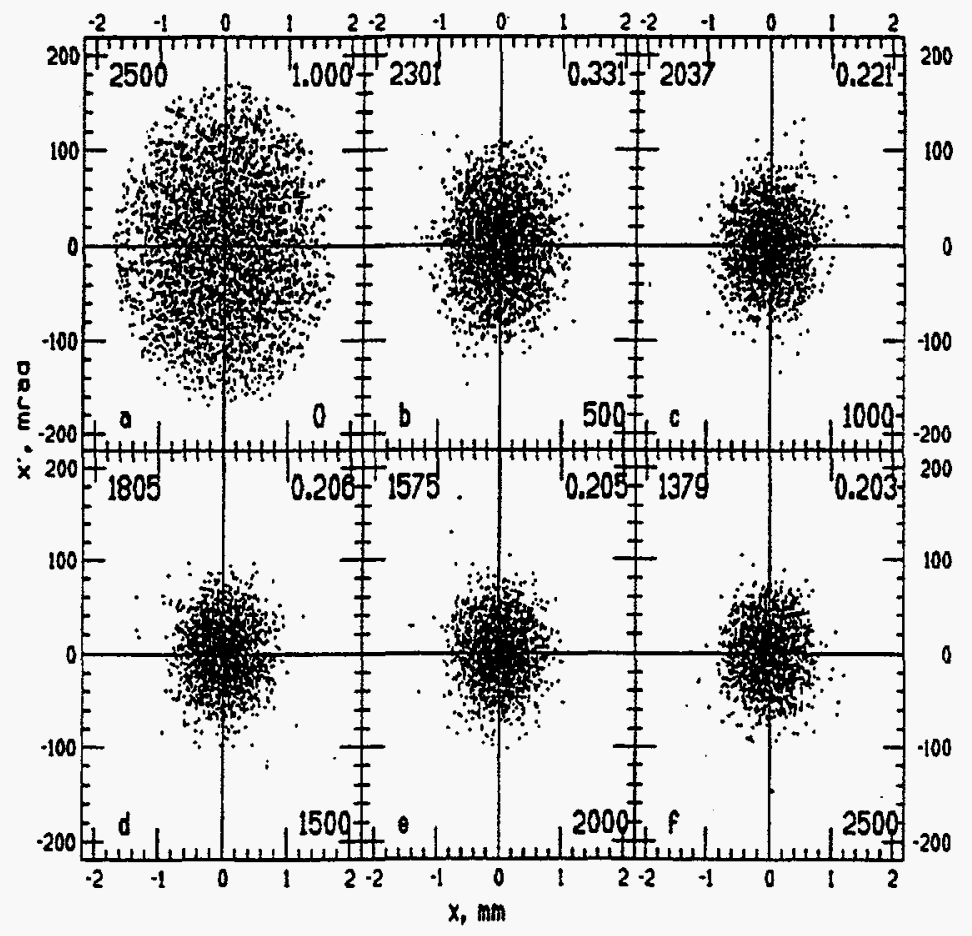

Figure 8: SIMUCOOL simulation of transverse emittance cooling in a storage ring 
$\mathrm{MeV} / \mathrm{c}$. The initial normalized transverse emittance was $1900 \mathrm{~mm} \mathrm{mr}$. The cooling achieved was $18 \%$, compared to $20 \%$ predicted by the rms equations. The cooling was found to be insensitive to the precise betatron function matching over the range from $10-12 \mathrm{~cm}$. The energy spread produced by the absorber had strong correlations with the particles amplitude and time.

A PARMELA simulation[25] of the minimum achievable transverse emittance using a 60 $\mathrm{cm}$ long rod of passive $\mathrm{Li}$ absorber inside a $7 \mathrm{~T}$ solenoid is shown in Fig. 9. The rod occupies longitudinal distances from 70 to $130 \mathrm{~cm}$. Cooling of the normalized emittance occurs for large values of the initial emittance. However, as the initial emittance is reduced, eventually the cooling just balances the heating and the emittance remains constant throughout the rod. This represents the case of minimum achievable emittance. If the initial emittance is reduced further, heating dominates and the normalized emittance increases in the Li.

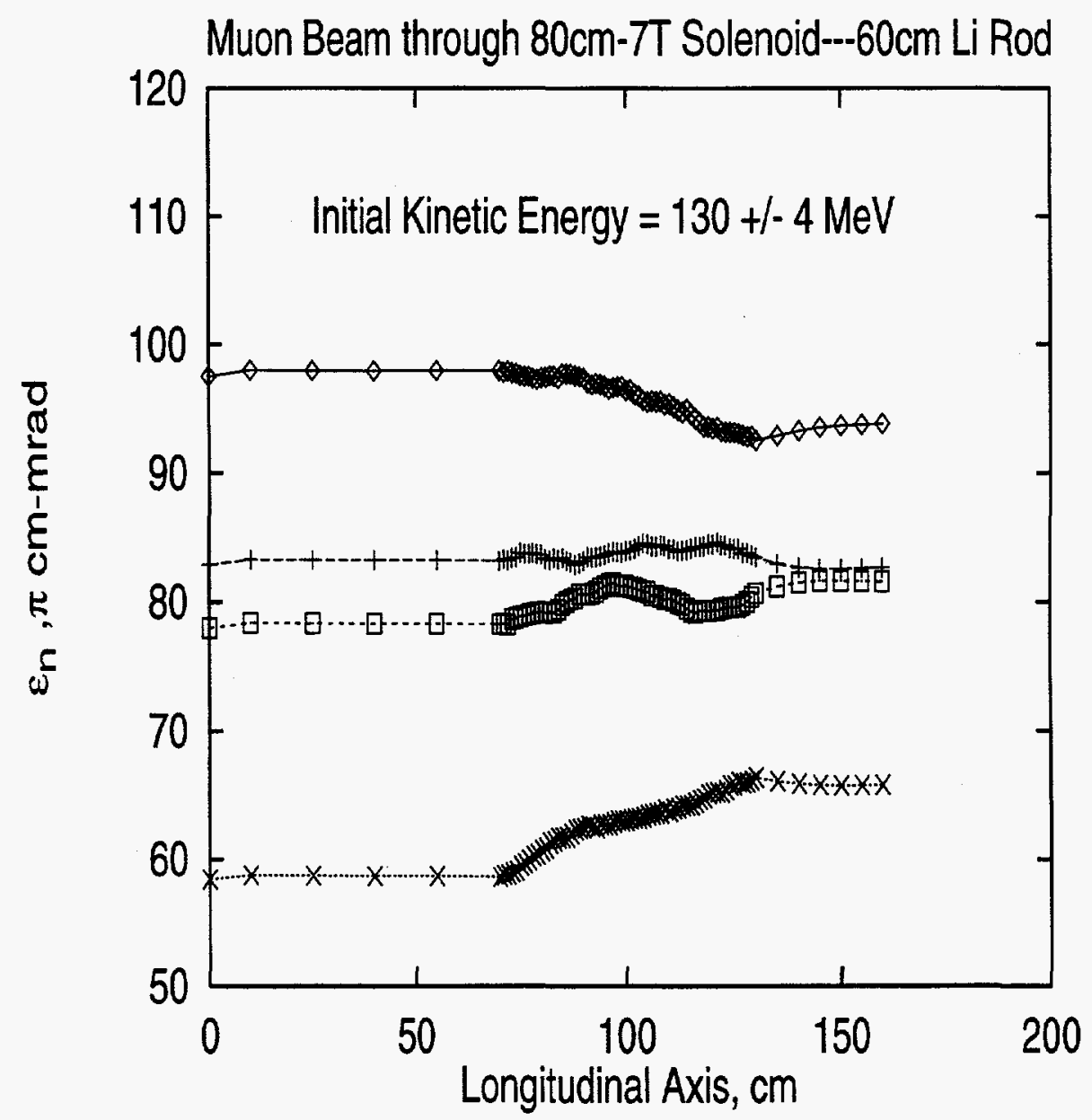

Figure 9: PARMELA simulation of transverse emittance cooling with a passive $\mathrm{Li}$ rod inside a solenoid 


\section{Scheme for Ionization Cooling}

\subsection{Overview}

For the muon collider we require a reduction of the normalized $\mathrm{x}$ and $\mathrm{y}$ transverse emittances by almost three orders of magnitude (from $\approx 15,000$ to $40 \mathrm{~mm} \mathrm{mr}$ ), and a reduction of the normalized longitudinal emittance by one order of magnitude [26]. This cooling is obtained in a series of cooling sections. Each of these sections contains some combination of the following elements:

- transverse cooling cells

- longitudinal cooling cells

- linear accelerators

- matching cells

The cooling section consists of alternating parts for transverse cooling, longitudinal cooling, and reacceleration. The cooling parameters are adjusted to provide the most efficient cooling for the beam conditions at that location. Matching cells must be provided to accept the beam from the phase rotation part of the accelerator. If induction linacs are used there, then it will probably be necessary to also use induction linacs in the early part of the cooling system. A matching section will be required to deliver the cooled beam to the first accelerator booster ring. In addition, matching cells (consisting of a pair of solenoids, for example) must be provided for the transition between the different parts of the cooling section. Throughout this process appropriate momentum compaction and $r f$ fields must be used to control the bunch in the presence of space charge, wake field, and resistive wall effects.

\section{Transverse emittance cooling}

Transverse cooling of the initial, large emittance muon beam is accomplished using FOFO cooling cells. These consist of a lattice of solenoids with alternating field directions and a short piece of absorber in the space between them. The solenoids provide a small $\beta_{\perp}$ in the absorbers. The requirement for efficient cooling is that the angle of the beam trajectory with the system axis inside the absorber caused by the focussing magnets must be large compared to the characteristic scattering angle in the material. Thus any practical design must be able to transmit a beam with a large angular acceptance. The preferred absorber material is LiH because it's higher density allows shorter lengths of absorber and better overlap with the regions of small $\beta_{\perp}$.

Near the end of the cooling sections, where the beam emittance is small, the FOFO solenoids may not provide sufficiently strong focusing. At this point current-carrying lithium rods might be necessary to produce the final transverse cooling. In this case the rod serves simultaneously to maintain the low $\beta_{\perp}$ and provide the required energy loss. Similar lithium rods, with surface fields of $10 \mathrm{~T}$, were developed at Novosibirsk and have been used as focusing elements at FNAL and CERN. It might be possible to use liquid lithium columns to raise the surface field to $20 \mathrm{~T}$ and improve the resultant cooling [27]. Another possibility is to cool the longitudinal emittance more than the required amount and then use reverse wedges in a dispersive region to exchange longitudinal for transverse emittance. 


\section{Longitudinal emittance cooling}

Longitudinal cooling is provided by using wedge-shaped absorbers in a dispersive region of the beamline. The lattice here consists of more widely spaced alternating solenoids with the dispersion introduced by using chicanes (series of dipoles). Dispersion must be introduced and removed by wedges in both the $\mathrm{x}$ and $\mathrm{y}$ dimensions.

\section{Cooling section accelerators}

Linear accelerators are used initially for bunch compression in order to minimize the amount of low frequency $r f$ required. In the cooling channel accelerators are used to restore the beam energy lost in the absorbers and wedges. It would be desirable, if practicable, to economize on linac sections by forming groups of stages into recirculating loops.

\subsection{Example Cooling Scenario}

Model examples of the cooling lattice have been designed that make use of a linear array of the cooling elements discussed in the previous section. The model discussed here contains all the essential components for achieving the emittance reduction required for the muon collider described in this document. However, it is incomplete since all the matching sections have not been specified, some higher order corrections and aberrations have not been considered and the performance has not been optimized. Computer codes for the analytic design of cooling scenarios and for particle tracking through all the elements of the cooling system are under active development. Tracking studies to check the performance of the analytic model are currently in progress. Given sufficient time and resources, it should be possible to produce a fully optimized cooling design.

The model design uses a FOFO lattice for initial focusing of the muon beam. Spherical aberrations due to solenoid end effects, wake fields, and second order $r f$ effects have not yet been included. The phase advance in each cell of the FOFO lattice is made as close to $\pi / 2$ as possible in order to minimize the $\beta_{\perp}$ at the location of the absorber, consistent with the phase spread caused by:

- the maximum space charge transverse defocusing

- a $3 \sigma$ fluctuation of momentum

- a $3 \sigma$ fluctuation in amplitude

Bending magnets are introduced to generate dispersion, but the dispersion is kept equal to zero at the center of all solenoids. The maximum allowed beam angle with respect to the axis, due to dispersion, is $67^{\circ}$. In the early FOFO stages, the solenoids are relatively large and their fields are limited to $13 \mathrm{~T}$. In later stages the transverse emittance is reduced using $\mathrm{Li}$ lenses with a surface field of $13 \mathrm{~T}$. The maximum bending fields used in the emittance exchange regions are 9.2 T. Except for the dispersive sections and the Li lenses, it is assumed that both charges will use the same channel.

The transverse and longitudinal emittances are shown in Fig. 10 as a function of section number, together with the beam energy. In the early sections, relatively strong wedges are used inside a FOFO lattice to rapidly reduce the longitudinal emittance, while the transverse emittance is reduced relatively slowly. The object here is to reduce the bunch length, thus 
allowing the use of higher frequency and higher gradient $r f$ in the reacceleration linacs. In the later sections, the emittances are reduced close to their asymptotic limits. The charges are separated for the Li lens sections at the end. During the final cooling the energy is allowed to fall to $15 \mathrm{MeV}$ in order to minimize the value of $\beta_{\perp}$ Some important properties of the cooling system based on the analytic calculations of the expected beam properties are summarized in Table 2.

\section{Magnets for the Muon Cooling System}

The muon cooling system consists of hundreds of superconducting solenoids that have central inductions ranging from 2 to 13 tesla. Solenoids can be found in both the cooling sections and the emittance exchange sections of the muon cooling system. Solenoids of two types are presented here; the cooling section solenoids and the emittance exchange solenoids. Both types of solenoids are relatively short compared to their coil inside diameter. Both types of solenoids in the muon cooling system will have a warm bore. The bore tube should be

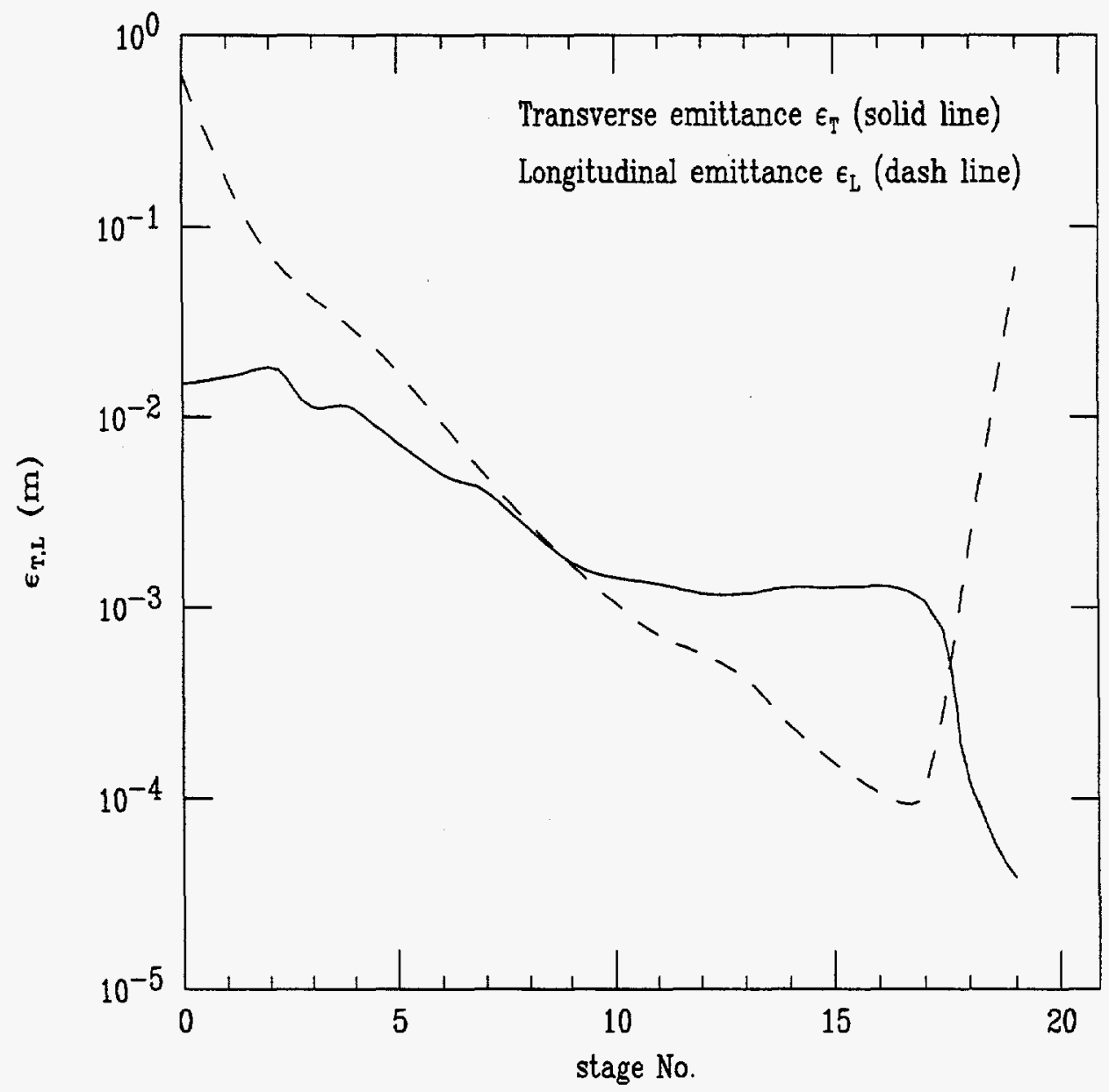

Figure 10: Normalized transverse and longitudinal emittances and muon energy as a function of section number in the model cooling system 
Table 2: Cooling section summary

\begin{tabular}{|c|c|c|c|}
\hline total length & & 743 & $\mathrm{~m}$ \\
\hline sections & & 19 & \\
\hline total acceleration & & 4.8 & $\mathrm{GeV}$ \\
\hline accelerator length & & 690 & $\mathrm{~m}$ \\
\hline$\mu$ decay loss & & 45 & $\%$ \\
\hline contingency loss & & 20 & $\%$ \\
\hline & Entrance & Exit & \\
\hline$\overline{\mathrm{KE}}$ & 300 & 15 & $\mathrm{MeV}$ \\
\hline $\mathrm{p}$ & 392 & 58 & $\mathrm{MeV} / \mathrm{c}$ \\
\hline$\beta$ & 0.966 & 0.481 & \\
\hline$\epsilon_{x N}(\mathrm{rms})$ & 15000 & 39 & $\mathrm{~mm} \mathrm{mr}$ \\
\hline$\epsilon_{z N}(\mathrm{rms})$ & 61.2 & 6.0 & $\mathrm{~m} \%$ \\
\hline$\sigma_{z}$ & 1.50 & 0.35 & $\mathrm{~m}$ \\
\hline$\frac{\delta p}{p}$ & 11.0 & 31.7 & $\%$ \\
\hline$\mu$ intensity & 7.5 & 3.0 & $10^{12} /$ bunch \\
\hline
\end{tabular}

cooled to remove the energy deposited from muon decay products. Muon decay products can deposit around a watt per meter into the warm bore tube that travels down through all of the magnets in the muon cooling channel.

The muon cooling system also has dipoles that are part of the emittance exchange sections of the cooling channel. We believe that the dipoles can be modified picture frame superconducting dipoles. This type of design was chosen because the length of the dipole is relatively short in comparison to the horizontal and vertical aperture. (In some cases the dipole length is shorter than the dipole gap.) The central induction of the dipoles is high enough to preclude the use of conventional water cooled dipoles.

\subsection{Muon Cooling Solenoids}

The cooling solenoids are close together, and they are powered with alternating polarity. This arrangement produces a zero field (on axis) at a point between the solenoids. In the low field region between the solenoids are pieces of lithium or beryllium to cool the muons. The arrangement of a representative cooling solenoid is shown in Fig. 11. A parameter set for the cooling section solenoids is presented in Table 3 for a representative set of solenoids. The cooling section solenoids get smaller as the muon beam emittance decreases. The central induction at the center of the solenoids goes up as one goes down the muon cooling channel.

The 5 and the 7 tesla solenoids can be fabricated from niobium titanium conductor that operates at $4.2 \mathrm{~K}$. At some point down the cooling channel, the solenoid induction becomes too large to be generated by niobium titanium at $4.2 \mathrm{~K}$. When the solenoid central induction reaches 11 tesla, the solenoid must be fabricated from an A-15 superconductor (multifilamentary niobium tin is the most commonly used A-15 conductor). Some of the solenoids that have a central induction between 7 tesla and 9.5 tesla can be fabricated from niobium titanium, but they must be cooled to $1.8 \mathrm{~K}$. The high field solenoids can be hybrid 
Table 3: Muon cooling solenoid magnet parameters for three sections in the muon cooling channel

\begin{tabular}{|c|c|c|c|}
\hline Parameter & & & \\
\hline Solenoid Induction* $(\mathrm{T})$ & 5.0 & 7.0 & 11.0 \\
\hline Solenoid Length (mm) & 531 & 386 & 131 \\
\hline Warm Bore Radial Aperture(mm) & 310 & 65 & 39 \\
\hline Solenoid Coil Inside Radius (mm) & 325 & 80 & 54 \\
\hline Solenoid Coil Outside Radius (mm) & 475 & 160 & 108 \\
\hline Solenoid Current Density** $\left(\mathrm{A} \mathrm{mm}^{-2}\right)$ & 53.2 & 83.2 & 274.1 \\
\hline Peak Induction in Coil ${ }^{* *}(\mathrm{~T})$ & 6.5 & 7.2 & 12.4 \\
\hline Solenoid Stored Energy ${ }^{* *}(\mathrm{~kJ})$ & 4752 & 290 & 163 \\
\hline Coil Superconductor & $\mathrm{Nb}-\mathrm{Ti}$ & $\mathrm{Nb}-\mathrm{Ti}$ & $\mathrm{Nb}_{3} \mathrm{Sn}$ \\
\hline Superconductor Temperature (K) & 4.2 & 4.2 & 4.2 \\
\hline
\end{tabular}

solenoids with small niobium tin solenoids inside larger niobium titanium outsert solenoids.

The 11 tesla solenoid described in Table 3 was assumed to be made from multifilamentary niobium tin in a copper based matrix. A conductor of this type would have up to 30 percent copper within the matrix. The coil current density appears to be reasonable, given the stored energy of the solenoid.

Since the solenoids operate with opposite polarity, there are large forces pushing the solenoids apart. It has been assumed that the solenoids can be separated by cold supports. Room temperature ports between the solenoid coils can be used to provide water cooling to the lithium or beryllium rods used to cool the muons. The solenoid sections can be fabricated so that several solenoid pairs are in a group. These solenoids would share common cryogenic cooling and perhaps a common power supply and leads

\subsection{Muon Emittance Exchange Solenoids}

The emittance exchange solenoids are further apart and they have the same polarity (in terms of solenoid performance, the polarity of the adjacent solenoid does not seem to make much difference). The parameter set for some representative emittance exchange solenoids is presented in Table 4. Like the cooling section solenoids, the emittance exchange solenoids get smaller as the muon beam emittance decreases. The central induction of the solenoids goes up as one goes down the cooling channel.

The 5 and the 7 tesla solenoids can be fabricated from niobium titanium conductor that operates at $4.2 \mathrm{~K}$. When the solenoid central induction reaches 11 tesla, the solenoid must be fabricated from an A-15 superconductor, such as niobium tin. The 11 tesla solenoid is quite short compared to its cold bore diameter. As a result, more current is needed in the coil to generate 11 tesla at the magnet center. The peak magnetic induction in the coil is quite high for this magnet. Operation of this magnet at $4.2 \mathrm{~K}$ may be marginal, but operation appears to be possible at $1.8 \mathrm{~K}$. Other A-15 materials, such as niobium titanium tin, may 
improve the margin at $4.2 \mathrm{~K}$. The high field solenoids can be hybrid solenoids with small niobium tin solenoids inside niobium titanium outsert solenoids.

The major problem with the muon emittance exchange system is the dipoles that are mixed with the solenoids. In order for the dipole field to be sharply defined, the dipoles must return their flux with an iron return path. The dipole iron return path may interfere with the solenoids that are near the dipoles. Individually, the solenoids can be built. The combination of dipoles and solenoids may be a problem. Considerable design work and a three dimensional magnetic field analysis are required before this problem can be completely solved. In some sections, quadrupole focusing of the muon beam may be desirable. Assuming that the dipoles and solenoids can be mixed, the forces between the two types of magnets can be taken up by a cold support between them.

\subsection{Muon Emittance Exchange Dipoles}

The emittance exchange dipoles bend the muon beam and spread it according to momentum. The dipoles that are in this part of the muon cooling system must have an aperture large

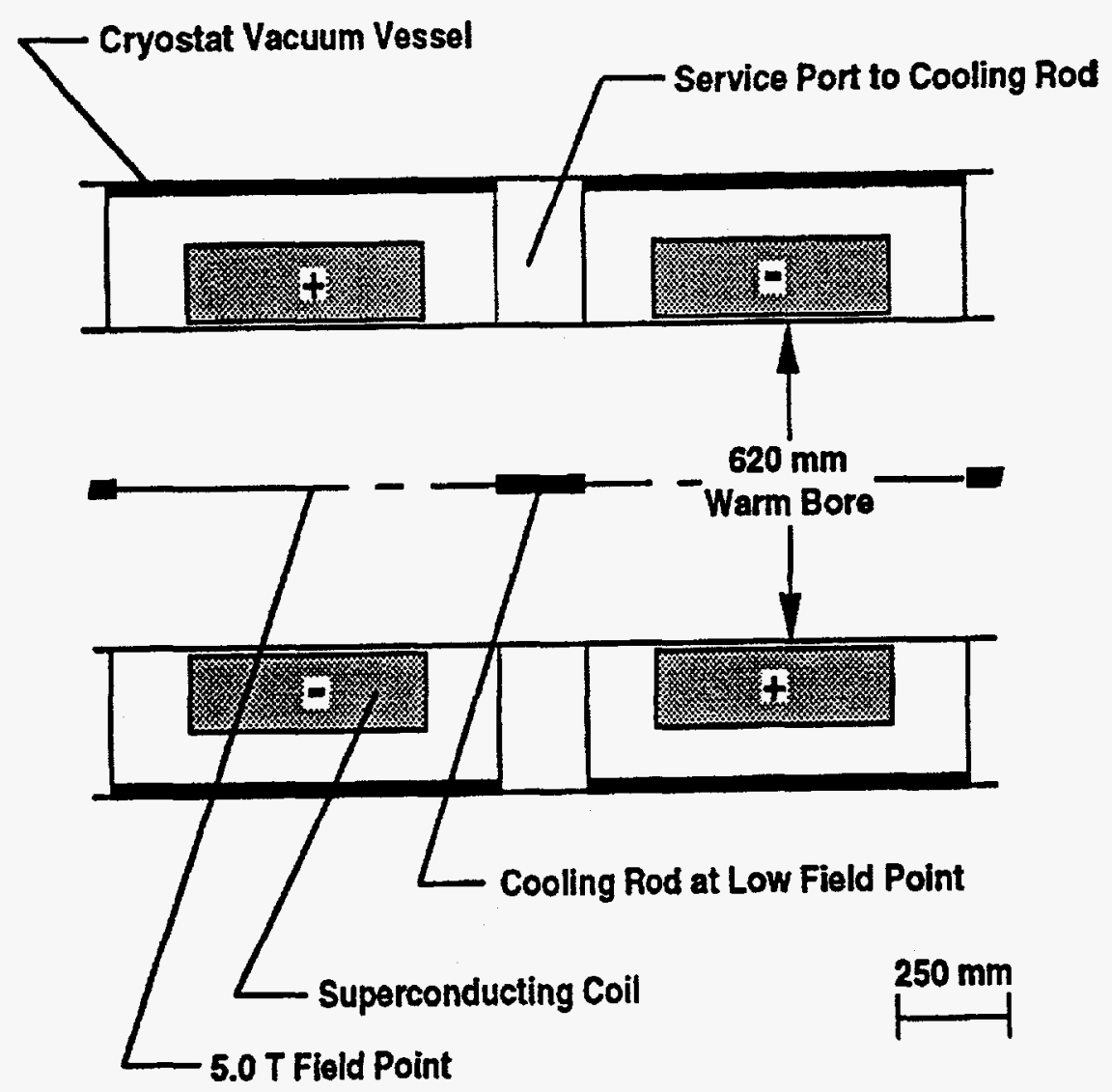

Figure 11: Cross-section of the superconducting solenoids of the muon cooling channel 
Table 4: Muon emittance exchange solenoid magnet parameters for three sections in the muon cooling channel

\begin{tabular}{|c|c|c|c|}
\hline Parameter & & & \\
\hline Solenoid Induction* $(\mathrm{T})$ & 5.0 & 7.0 & 11.0 \\
\hline Solenoid Length (mm) & 586 & 281 & 62 \\
\hline Warm Bore Radial Aperture (mm) & 309 & 75 & 19 \\
\hline Solenoid Coil Inside Radius (mm) & 325 & 90 & 34 \\
\hline Solenoid Coil Outside Radius (mm) & 400 & 170 & 88 \\
\hline Solenoid Current Density** $\left(\mathrm{A} \mathrm{mm}^{-2}\right)$ & 43.3 & 93.4 & 374.8 \\
\hline Peak Induction in Coil ${ }^{* *}(\mathrm{~T})$ & 6.0 & 7.5 & 13.9 \\
\hline Solenoid Stored Energy/Cell ${ }^{* *}(\mathrm{~kJ})$ & 4130 & 290 & 120 \\
\hline Coil Superconductor & $\mathrm{Nb}-\mathrm{Ti}$ & $\mathrm{Nb}-\mathrm{Ti}$ & $\mathrm{Nb}_{3} \mathrm{Sn}$ \\
\hline Superconductor Temperature (K) & 4.2 & 4.2 & 4.2 \\
\hline
\end{tabular}

enough to transport the muon beam and the sagitta of the bent beam. Early in the muon cooling process, these dipoles are large. They bend the muon beam over angles approaching 60 degrees. As the muon emittance is reduced, the vertical aperture of the dipoles is reduced. The horizontal aperture still has the bent beam sagitta, but it is also reduced to a great extent. The bend angle does go down somewhat as one proceeds down the cooling channel.

Table 5 presents the design parameters for a representative set of emittance exchange dipoles. At the start of muon cooling the vertical beam diameter is enormous. As a result, the dipole length to vertical aperture ratio is less than one. This means that the effective length of the dipole is quite a bit longer than its physical length. As the muon emittance is reduced, the dipole gap gets smaller. Unfortunately, the dipóle length also gets shorter, so the length to gap ratio is still around one. Because, the length to aperture ratio is around one, conventional superconducting dipole designs cannot be employed. A modified picture frame dipole design is proposed because this type of dipole will behave like a short conventional room temperature copper and iron dipole. The proposed magnet would be superconducting and it would be similar to magnets that have been proposed for compact light sources and small electron storage rings. Figures 12 shows a cross-sectional view of a possible dipole.

The dipoles shown in Table 5 appear to be feasible in and of themselves. The magnetic field will extend beyond the ends of the magnet by about a quarter of a gap (in the way conventional copper and iron dipoles behave). The iron in the dipole would be cold. The dipole might share a common cryostat with other elements in the emittance exchange magnet string. The dipoles and the adjacent solenoids would all have a warm bore. The elements for reducing the energy of high momentum muons would also be at room temperature. The problem with the dipoles is the fact that they share space with some high field superconducting solenoids that have an aperture comparable to the dipoles. The space between the dipoles and adjacent solenoids is an important issue. This space may have to be increased or a magnetic shield may have to be used. 
Table 5: Muon emittance exchange dipole magnet parameters for three sections in the muon cooling channel

\begin{tabular}{llll}
\hline \hline \multicolumn{1}{c}{ Parameter } & & & \\
\hline Dipole Average Induction (T) & 1.75 & 2.13 & 3.25 \\
\hline Dipole Bend Angle (deg) & 59 & 50 & 40 \\
\hline Dipole Iron Length (mm) & 600 & 290 & 66 \\
\hline Dipole Horizontal Half Aperture (mm) & 400 & 119 & 36.5 \\
\hline Dipole Vertical Half Aperture (mm) & 319 & 85 & 29 \\
\hline Dipole Cold Iron Height (mm) & 1440 & 460 & 190 \\
\hline Dipole Cold Iron Width (mm) & 1610 & 560 & 250 \\
\hline Dipole Stored Energy (kJ) & $\sim 370$ & $\sim 23$ & $\sim 1.9$ \\
\hline Dipole Coil Superconductor & $\mathrm{Nb}-\mathrm{Ti}$ & $\mathrm{Nb}-\mathrm{Ti}$ & $\mathrm{Nb}-\mathrm{Ti}$ \\
\hline \hline
\end{tabular}

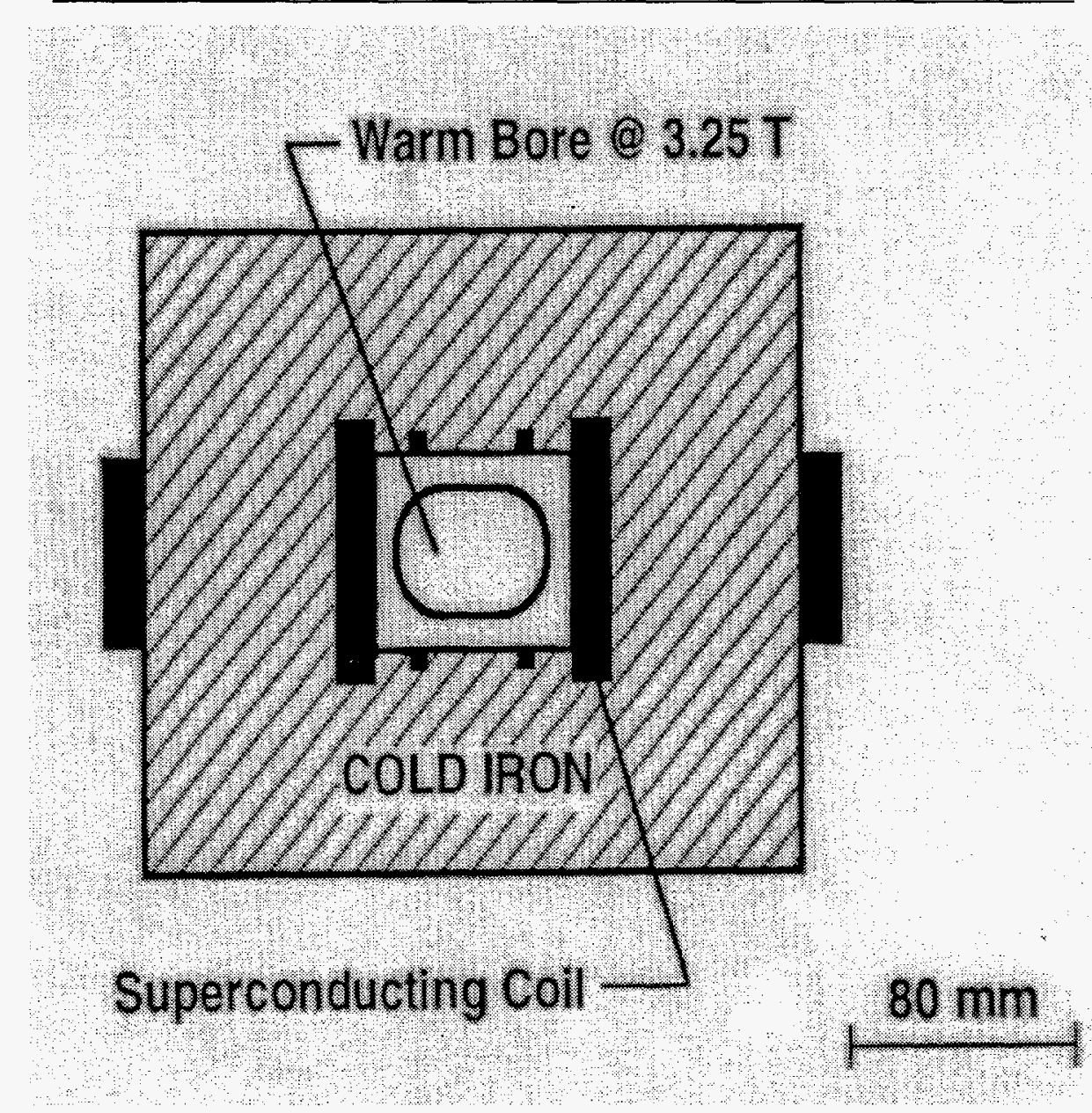

Figure 12: Cross-section of muon emittance exchange dipole of the muon cooling system

\section{A Possible Experimental Demonstration}

A BNL-CEBAF-Fairfield-Michigan-Stony Brook-Yale collaboration has submitted a letter of intent[28] to the AGS to build a prototype cooling cell with properties as close as possible 
to those required in a muon collider. The goal would be to measure the performance of the device and compare it with predictions from the Monte Carlo programs that are being used to design the collider. Since the performance of the cooling section plays an essential role in achieving the required luminosity and the cooling section may represent a significant fraction of the total cost of a muon collider, it is essential that the design Monte Carlo accurately model the achievable cooling. The experiment would also check that muon losses in the focusing fields and absorbing materials do not exceed the predicted amounts.

The initial phase of the proposed experiment would use a cylindrical piece of lithium, 12 $\mathrm{cm}$ in radius and $60 \mathrm{~cm}$ in length as the cooling rod. A solenoid with a maximum central field strength of $7 \mathrm{~T}$ would focus the muons in the interior of the rod. The absorber material would be replaced at some point with different lengths of absorber and with $\mathrm{Be}$ or $\mathrm{LiH}$ absorbers in order to further check the accuracy of the Monte Carlo predictions.

In order to simulate the conditions in the muon collider as closely as possible, the momentum of the incident muon should be around $200 \mathrm{MeV} / \mathrm{c}$. For a $0.6 \mathrm{~m}$ long lithium rod the final momentum would be around $140 \mathrm{MeV}$. The focusing in the rod should be as strong as possible, since Eq. 14 shows that the minimum emittance that can be reached is proportional to $\beta_{\perp}$.

The layout of the proposed experiment is shown in Fig. 13.

A large, diffuse beam of muons passes through a hadron absorber and enters an approximately $6 \mathrm{~m}$ long experimental area. The dipole D1 together with the position measuring detectors P1-5 are used to measure the momentum of each incoming track. A fraction of the beam will be captured by the solenoid magnet $\mathrm{S} 1$ and focused into an absorber. The momentum of the muons leaving the solenoid is measured using the spectrometer consisting of dipole magnet D2 and detectors P6-10. Trigger counters would tag tracks entering the absorber. A particle identification system would be necessary to identify pion and electron background in the muon beam. The dipole D1 would have to be adjusted to examine a series of different central values for the incident momentum. The momentum spread could be determined by software selection of the tracks used in the analysis.

In the following we consider an example case with an initial normalized emittance of $4000 \mathrm{~mm}-\mathrm{mr}$. The beam has a $1 \sigma$ radius of about $3 \mathrm{~cm}$ inside the lithium. The dipoles have central field values around $0.3 \mathrm{~T}$ and have widths and lengths appropriate to existing 72D18 magnets at the AGS. We assume a bend angle in the dipoles of $0.2 \mathrm{rad}$.

PARMELA results for the change in transverse normalized emittance as a function of axial distance $\mathrm{z}$ are shown in Fig. 14 and summarized in Table 6

Under the conditions listed in Table 6, the cooling rate is always larger than the heating rate and the normalized emittance of the beam leaving the rod is smaller than the normalized emittance entering it. However, the geometric emittance increases after traversing the rod. In an actual muon collider this stage must be followed by a reacceleration section that restores the starting momentum in order to get cooling of the geometric emittance.

One of the design requirements for the experiment is the ability to measure individual muon tracks. This will permit software "control" of the effective cooling rate. Software selection of initial beam tracks will allow us to reconstruct final beam emittance as a function of initial beam emittance. The experiment can be reanalyzed for smaller initial transverse emittances. As the initial emittances decrease, the cooling in the lithium becomes less effective relative to the heating and the net decrease in normalized emittance also drops. As 
Table 6: Transverse emittance cooling experiment

\begin{tabular}{llll}
\hline \hline & IN & OUT & \\
\hline $\mathrm{KE}$ & 130 & $70 \mathrm{MeV}$ & \\
\hline $\mathrm{p}$ & 211 & 140 & $\mathrm{MeV} / \mathrm{c}$ \\
\hline$\beta$ & 0.894 & 0.798 & \\
\hline$\gamma$ & 2.23 & 1.66 & \\
\hline$\epsilon_{x N}$ & 4000 & 3100 & $\mathrm{~mm} \mathrm{mr}$ \\
\hline$\epsilon_{x}$ & 2006 & 2340 & $\mathrm{~mm} \mathrm{mr}$ \\
\hline$\beta_{\perp}$ & 20.1 & 13.3 & $\mathrm{~cm}$ \\
\hline$\sigma_{x}$ & 20 & 18 & $\mathrm{~mm}$ \\
\hline$\sigma_{x^{\prime}}$ & 100 & 133 & $\mathrm{mr}$ \\
\hline
\end{tabular}

we saw in Fig. 9, this configuration should still produce net cooling until the initial emittance reaches about $820 \mathrm{~mm}-\mathrm{mr}$. These measurements are important since they demonstrate the

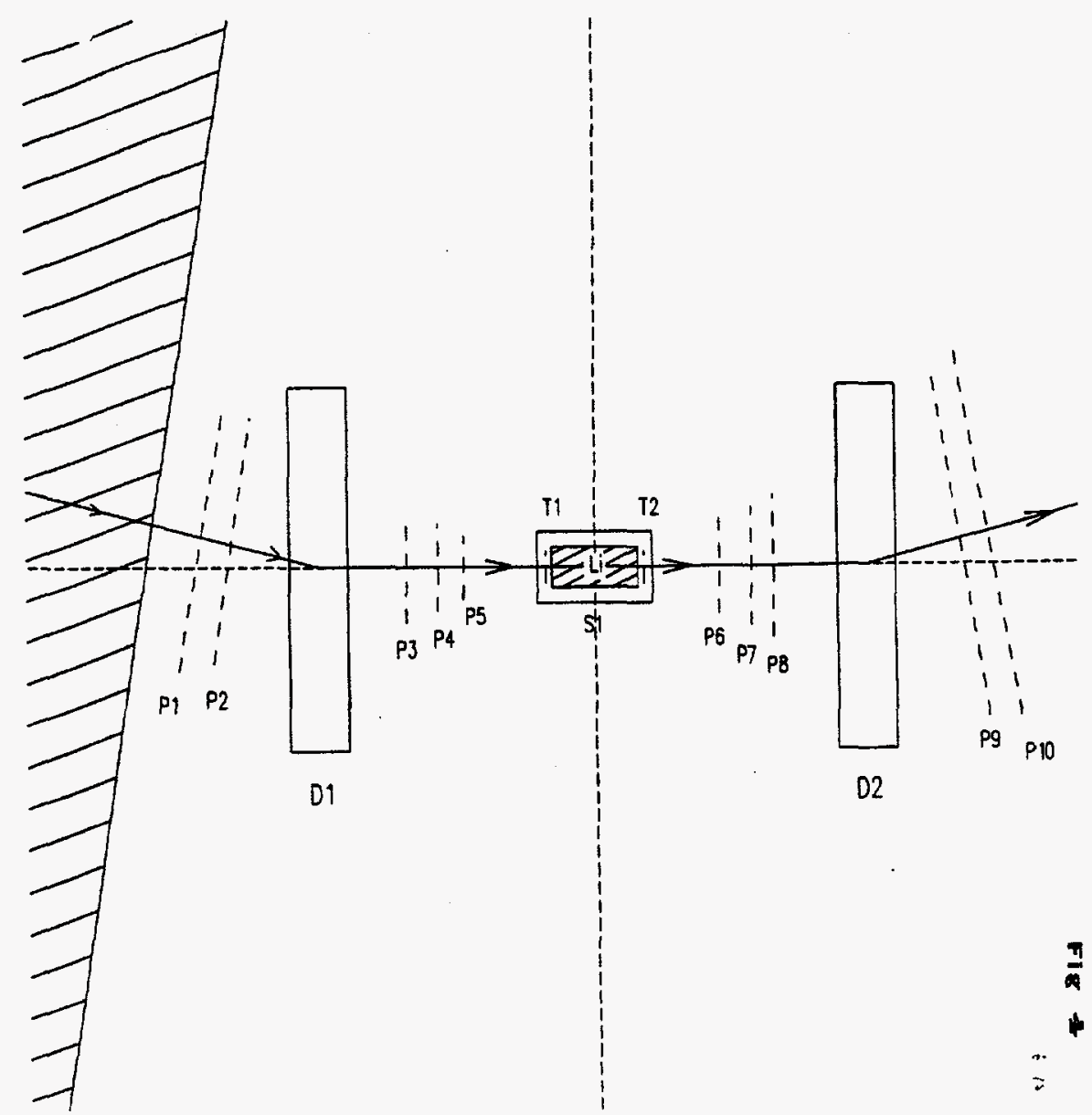

Figure 13: Layout of possible AGS experiment to test ionization cooling of transverse emittance 


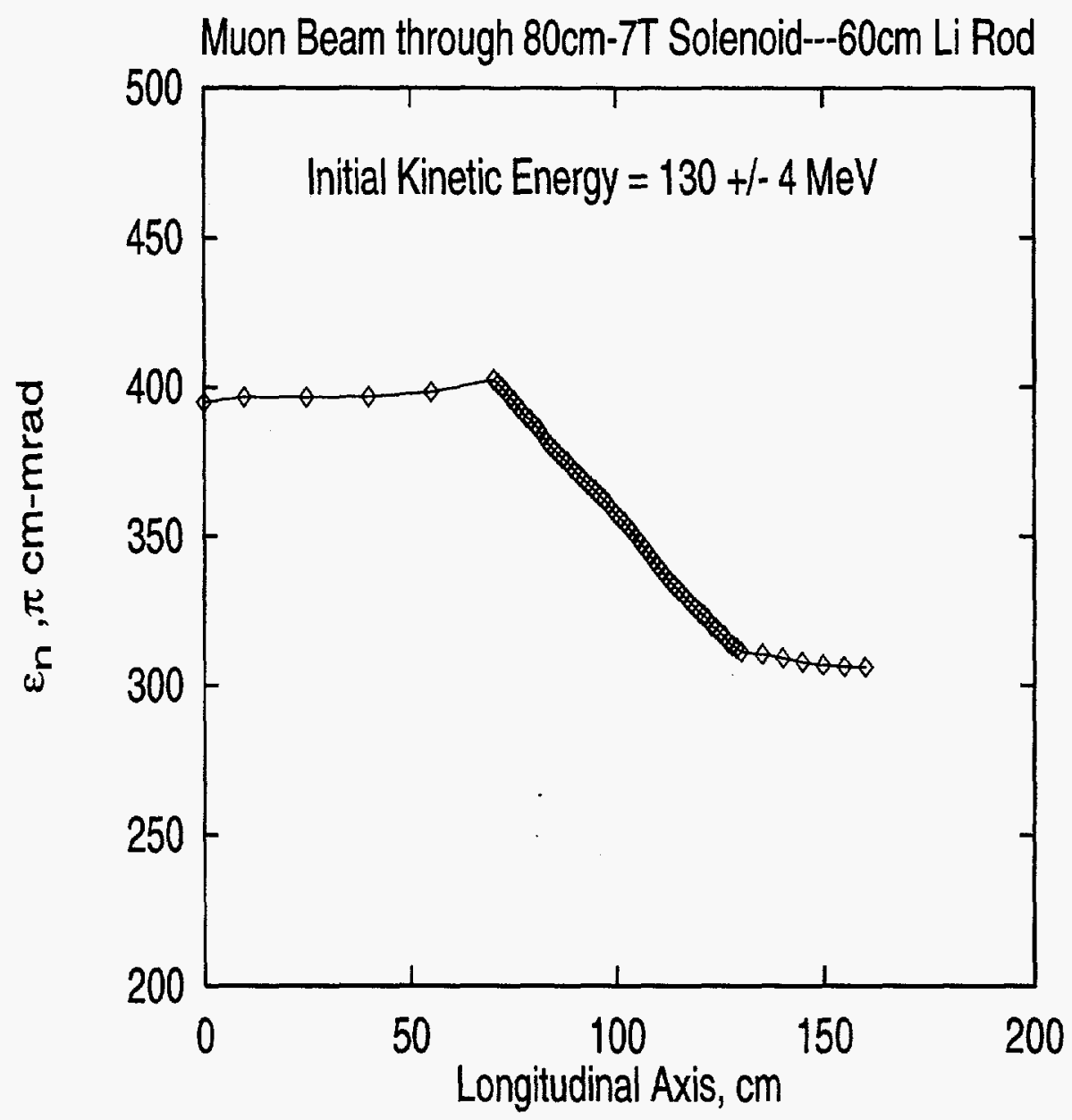

Figure 14: PARMELA simulation of transverse emittance in the AGS cooling experiment

minimum emittance achievable for a given field strength and absorber length.

The experimental test of cooling would require accurately measuring the position, angle, and momentum of individual muon tracks before and after the rod in order to measure the initial and final emittances. Since the chambers are likely to be in the strong fringe field of the solenoid, a good field map will be required. We need at least three $\mathrm{x}$ and $\mathrm{y}$ measuring planes on each side of the rod to measure the curvature of the track. The six chambers closest to the rod require measurements with moderate resolution $(\approx 300 \mu \mathrm{m})$. The expected fractional change in normalized emittance for the case described in Table 6 is about $23 \%$. In order to measure the smaller effects expected with lower initial emittances, we would like to determine $\Delta \epsilon_{x N} / \epsilon_{x N}$ to an accuracy of $\approx 0.1 \%$.

In order to measure individual tracks we propose using the slow extracted beam from the AGS. The useful muon rate should be larger than $\approx 10^{2}$ per spill so that a complete measurement can be made in about an hour, in order to minimize the effects of systematic errors. If the useful rate is smaller than $\approx 10^{4}$ per spill, it should be possible to use a fairly simple data acquisition system. 


\section{Summary}

The process of ionization cooling offers a method for reducing the 6-dimensional normalized emittance of the muon beam by a factor of $\approx 10^{6}$. A simple analytic theory has been developed that demonstrates the dependence of the net cooling on various experimental parameters. The simple theory has been checked and realistic arrangements have been examined using Monte Carlo simulations. Transverse cooling of the initial beam can be achieved using passive $\mathrm{Li}$ or $\mathrm{Be}$ absorbers in a FOFO lattice or a solenoid channel. The last factor of 10 in transverse cooling probably requires the use of current-carrying $\mathrm{Li}$ lenses. Efficient longitudinal cooling requires the use of wedge shaped absorbers in a dispersive section of the beam line. An example, multi-stage cooling scenario has been developed that meets the requirements of the muon collider using a mixture of FOFO lattice, $\mathrm{Li}$ lenses, wedge absorbers, $r f$ accelerators, and matching sections. Preliminary designs have been made of solenoids for use in the FOFO lattice and of solenoids and dipoles for use in the emittance exchange sections. Detailed simulation work, further optimization, and preparations for experimental demonstrations of critical components are currently in progress.

\section{ACKNOWLEDGEMENTS}

This research was supported by the U.S. Department of Energy under Contracts No. DE-AC02-76-CH00016, DE-AC02-76-CH03000 and DE-AC03-76-SF00098.

\section{References}

[1] Muon-Muon Collider: Feasibility Study, BNL report 52503, Fermilab report 96/092, LBNL report 38946, 1996.

[2] A.N. Skrinskii \& V.V. Parkhomchuk, Methods of cooling beams of charged particles, Sov.J.Part.Nucl. 12:223-247, 1981; V.V. Parkhomchuk \& A.N. Skrinsky, Ionization cooling: physics and applications, Proc. 12th Int. Conf. High Energy Phys., 1983, p. 485-7.

[3] D. Neuffer, Principles and applications of muon cooling, Proc. 12th Int. Conf. High Energy Phys., 1983, p. 481-4; D. Neuffer, Principles and applications of muon cooling, Part. Acc. 14:75-90, 1983.

[4] A.N. Skrinsky, Ionization cooling and muon collider, in J. Gallardo(ed), Proc. Workshop on Beam dynamics and technology issues for $\mu^{+} \mu^{-}$colliders, AIP Conf. Proc. 372, 1996, p. 133-9.

[5] R.C. Fernow \& J.C. Gallardo, Validity of the differential equations for ionization cooling, in D. Cline(ed), Physics Potential and Development of $\mu^{+} \mu^{-}$Colliders, Second workshop, AIP Conf. Proc. 352, 1995, p.170-7.

[6] B. Rossi, High Energy Particles, Prentice-Hall, 1952. 
[7] U. Fano, Penetration of protons, alpha particles, and mesons, Ann. Rev. Nuc. Sci. 13:1-66, 1963, eqn. 72.

[8] R.C. Fernow \& J.C. Gallardo, Muon transverse ionization cooling: stochastic approach, Phys.Rev.E 52:1039-42, 1995.

[9] T. Vsevolozhskaya, Kinetics of ionization cooling of muons, in J. Gallardo(ed), Proc. Workshop on Beam dynamics and technology issues for $\mu^{+} \mu^{-}$colliders, AIP Conf. Proc. 372, 1996, p. 159-167; T. Vsevolozhskaya, Ionization cooling of muons by strong focusing with a field of longitudinal current, Nuc. Inst. Meth. A 374:281-5, 1996.

[10] J. Gallardo, Useful formulae for uniform solenoid channel, Center for Accelerator Physics, BNL, $\mu \mu$ Tech. note 7-95.

[11] H. Wiedemann, Particle Accelerator Physics: basic principles and linear beam dynamics, Springer-Verlag, 1993, p. 187-8.

[12] D. Carey, The optics of charged particle beams, Harwood, 1987, p. 210.

[13] B. Bayanov et al., A lithium lens for axially symmetric focusing of high energy particle beams, Nuc. Instr. Meth. 190:9-14, 1981.

[14] G. Dugan et al, Mechanical and electrical design of the Fermilab lithium lens and transformer system, IEEE Trans. NS 30:3660-2, 1983.

[15] V. Balbekov, Achievable transverse emittance of beam in muon collider, in J. Gallardo(ed), Proc. Workshop on Beam dynamics and technology issues for $\mu^{+} \mu^{-}$colliders, AIP Conf. Proc. 372, 1996, p. 140-145.

[16] A. Van Ginneken, Fluctuations of muon energy loss and simulation of ionization cooling, Nuc.Instr.Meth.A 362:213-23, 1995.

[17] Particle Data Group, Review of Particle Properties, Phys. Rev. D 50:1173-1826, 1994.

[18] L. Landau, On the energy loss of fast particles by ionization, in D. ter Haar (ed), L.D. Landau, Collected Papers, Pergamon, 1965.

[19] GEANT manual, v. 3.2.1, CERN program library W5013, 1994.

[20] P. Vavilov, Ionization losses of high energy heavy particles, Sov. Phys. JETP 5:749, 1957.

[21] H. Bethe, Moliere's theory of multiple scattering, Phys. Rev. 89:1256-66, 1953.

[22] J.D. Jackson, Classical Electrodynamics, Wiley, 1962.

[23] Simulation work with tracking codes that has demonstrated ionization cooling include A. Van Ginneken (SIMUCOOL), R. Thun (private communication), R. Palmer \& J. Gallardo (MUMC), H. Kirk (PARMELA), and R. Fernow (ICOOL). 
[24] A. Van Ginneken \& D. Neuffer, private communications, February 1996.

[25] Los Alamos Accelerator Code Group, PARMELA; the code was modified by H. Kirk to include ionization loss and multiple scattering.

[26] R. Palmer et al, Muon Collider Design, in Proc. of the Symposium on Physics Potential and Development of Muon-Muon Colliders, San Francisco, CA, 1995.

[27] G.I. Silvestrov, Lithium lenses for muon colliders, in J. Gallardo(ed), Proc. Workshop on Beam dynamics and technology issues for $\mu^{+} \mu^{-}$colliders, AIP Conf. Proc. 372, 1996, p. $168-177$.

[28] R. Fernow et al., Possible demonstration of ionization cooling using absorbers in a solenoidal field, in J. Gallardo (ed), Proc. Workshop on Beam dynamics and technology issues for $\mu^{+} \mu^{-}$colliders, AIP Conf. Proc. 372, 1996, p. 159-167. Some earlier ideas on a demonstration experiment are described in R.C. Fernow et al., A possible ionization cooling experiment at the AGS, in D. Cline(ed), Physics Potential and Development of $\mu^{+} \mu^{-}$Colliders, Second workshop, AIP Conf. Proc. 352, 1995, p.155-169. 